\title{
Médiévales
}

Langues, Textes, Histoire

65 | automne 2013

Le couple dans le monde franc

\section{Éclipse ou Apocalypse. Remarques autour du nœud du dragon dans les miniatures des Commentaires de l'Apocalypse de Beatus de Liébana}

Eclipse or Apocalypse. On the Dragon Knot in the Miniatures of the Beatus of Liébana's Commentaries of the Apocalypse

\section{Anna Caiozzo}

\section{OpenEdition}

\section{Journals}

Édition électronique

URL : https://journals.openedition.org/medievales/7121

DOI : 10.4000/medievales.7121

ISSN : $1777-5892$

\section{Éditeur}

Presses universitaires de Vincennes

\section{Édition imprimée}

Date de publication : 1 décembre 2013

Pagination : 125-154

ISBN : 978-2-84292-396-9

ISSN : 0751-2708

\section{Référence électronique}

Anna Caiozzo, «Éclipse ou Apocalypse. Remarques autour du nœud du dragon dans les miniatures des Commentaires de l'Apocalypse de Beatus de Liébana », Médiévales [En ligne], 65 | automne 2013, mis en ligne le 20 janvier 2014, consulté le 22 avril 2022. URL : http://journals.openedition.org/ medievales/7121; DOI : https://doi.org/10.4000/medievales.7121 
Médiévales 65, automne 2013,p. 125-154

Anna CAIOZZO

\author{
ÉCLIPSE OU APOCALYPSE, REMARQUES AUTOUR \\ DU NQEUD DU DRAGON DANS LES MINIATURES \\ DES COMMENTAIRES DE L'APOCALYPSE DE BEATUS DE LIÉBANA
}

Le moine asturien Beatus, du monastère Saint-Martin de Liébana, rédigea dans la deuxième moitié du VIII ${ }^{\mathrm{e}}$ siècle deux commentaires importants sur l'Apocalypse de Jean et de Daniel' ${ }^{1}$. Ses textes furent en majorité enluminés à partir $\mathrm{du} \mathrm{IX}^{\mathrm{e}}$ siècle dans le Nord de la péninsule ibérique, dans les royaumes chrétiens du León et des Asturies.

Plusieurs exemplaires de ce cycle d'illustrations comportent un étrange motif stylistique qui, en d'autres occasions, passerait inaperçu, si sa symbolique dans le contexte de l'art musulman n'était pas aussi marquée. Certains Beatus présentent en effet une particularité iconographique dans la représentation de la Babylone aux serpents ${ }^{2}$ ou du Dragon aux sept têtes de l'Apocalypse, et de la Femme: il s'agit d'un nœud en forme de cœur dans le corps du dragon correspondant et, à première vue, dû à l'une de ses circonvolutions ; au demeurant, le dragon présente un caractère très ophidien.

Ce motif, le nœud en forme de cœur, n'est présent que dans onze des vingt-six manuscrits et fragments du cycle des Beatus, et totalement absent des

1. Je remercie David Juste et Philippe Morel pour leur relecture et leurs conseils. Le XVII canon du Concile de Tolède en 633, présidé par Isidore de Séville, rendit obligatoire la lecture de l'Apocalypse lors des offices entre Pâques et la Pentecôte. En effet, jusque-là, les Commentaires de l'Apocalypse étaient canoniques en Orient et inclus dans la liturgie, mais non en Occident.

2. Dans son étude sur l'iconographie de la Babylone aux serpents, N. Mezoughi n'avait visiblement pas remarqué le nœud décorant le corps du dragon: N. MezoughI, «La place de Babylone entourée de serpents entre L'Apocalypse et Le Livre de Daniel dans le Beatus », dans J. CABANOT éd., Millénaire de l'Abbaye, colloque international, 27 mai 1985, Mont-de-Marsan, 1986, p. 287-315. 
miniatures des bibles mozarabes ou médiévales européennes datant de la même époque et représentant des scènes de l'Apocalypse ${ }^{3}$.

Ce nœud apparaît dans plusieurs miniatures où le dragon (ou le serpent) figure comme être nuisible ou, mieux, comme l'Antéchrist lui-même ou ses séides: celle de la Femme vêtue de Soleil menacée par le dragon (Apoc. XII 1-18); scène d'adoration de la Bête (Apoc. XIII 1-10) et des démons en forme de grenouilles (Apoc. XVI 13-16), dans la scène de Satan enchaîné aux enfers avec sa créature (Apoc. XX, 1-3), et dans la Babylone aux serpents (Daniel $\mathrm{I}: 1 ; \mathrm{XIV} / 23)$.

\section{Origines et signification astrologiques et magiques du dragon}

Négativement connoté en Occident latin, associé aux forces du Mal, le dragon est une créature bien connue en Orient où, s'il renvoie en partie à des valeurs négatives, il appartient entre autres à la cosmologie, à la religion, à l'emblématique des dieux et des peuples préislamiques ${ }^{4}$. À l'époque islamique, il est perçu comme le protagoniste de choix des héros de l'épopée persane. Il devient au $\mathrm{IX}^{\mathrm{e}}$ siècle un concept astrologique et, à partir du XII ${ }^{\mathrm{e}}$ siècle, un thème décoratif fréquent dans l'aire syro-anatolienne qui, au demeurant, est un milieu où il est connu depuis les périodes les plus reculées.

Les dragons appartiennent en effet à la plupart des mythologies procheorientales depuis les époques les plus éloignées; certains sont des dragons originels illustrant les forces du chaos des premiers temps, tel le monstre marin Tiamat, un dragon combattu par le dieu Mardouk dont le symbole est aussi un dragon ${ }^{5}$. Le dragon est habituellement associé aux forces obscures, souterraines, maléfiques et porteuses de mort, à l'image d'Humbaba, monstre ou dragon combattu par Gilgamesh. Dans d'autres mythologies, chez les Zoroastriens d'Iran par exemple, le dragon, emblème du Mal, créature d'Ahriman ${ }^{6}$, est l'adversaire

3. Voir W. Neuss, Die katalanische Bibelillustration um die Wende des ersten Jahrtausends und die altspanische Buchmalerei, Bonn/Leipzig, 1922. P. K. KLEIN, «Les cycles de l'Apocalypse du haut Moyen Âge», dans Y. CHriste éd., L'Apocalypse de Jean, traditions exégétiques et iconographiques, III -XIII siècle, Actes du colloque de la fondation Hardt, 29 février-3 mars 1976, Genève, 1979, p. 135-186.

4. On peut voir S. KueHn, The Dragon in Medieval East Christian and Islamic Art, Leyde, 2011, qui fait l'état d'une partie des travaux menés sur le thème dans cette aire géographique et expose quelques thématiques sur le dragon.

5. T. JACOBSEN, «The Battle between Marduk and Tiamat», Journal of the American Oriental Society, 88 (1968), p. 104-108.

6. Sur le symbolisme des créatures nuisibles, M. Moazami, «Evil Animals in the Zoroastrian Religion», History of Religions, 44/4 (2005), p. 310-311 et, dans l'art musulman, A. CAIOzzo, «Autour des dragons célestes: astronomie, astrologie, magie et imaginaire en Orient médiéval», 
de prédilection des divers héros que Firdawsî célèbre au $\mathrm{x}^{\mathrm{e}}$ siècle dans sa grande épopée des rois, le Shâh Nâmeh ${ }^{7}$.

Pourtant, le dragon est aussi l'animal attribut de certains dieux mésopotamiens ${ }^{8}$ et, dans certains cas, sa présence est symbole de protection de la nature et des populations contre les calamités ${ }^{9}$. En Arménie, le dragon est vénéré, dès l'âge de pierre, sous le nom de vishap, associé à un culte des eaux ${ }^{10}$, et plus particulièrement dans les régions où l'homme doit irriguer la terre par des systèmes de canaux. Il est une sorte de divinité protectrice des eaux et des cultures. Le dragon est donc un animal mythique ambigu, craint et redouté, et de ce fait utilisé comme apotropaion.

Le thème du dragon connaît un renouveau particulièrement marqué dans l'art architectural et dans les arts mineurs musulmans (métaux, miniatures) lors de l'installation des Saljoukides en Anatolie. Aussi, certains historiens d'art ont-ils attribué la fréquence de ce thème dans l'art islamique de la haute Mésopotamie à la réminiscence d'un ancien symbole totémique des tribus turques désormais sédentarisées ${ }^{11}$. D'un certain point de vue, cela est exact, car les tribus turques avaient de surcroît longtemps côtoyé la culture chinoise qui se démarque du Proche-Orient par son intérêt pour les dragons perçus sous un jour favorable ${ }^{12}$.

dans F. Chen, T. Honegger éd., Good Dragons Are Rare: An Inquiry into Literary Dragons East and West, Francfort, 2009, p. 419-439.

7. Outre les dragons, le roi maudit Aži Dahaka, «monstre à trois gueules et à trois têtes et à six yeux », transformé en créature hybride par le mal et qui dévore ses sujets; déchu, puis enchaîné par le héros Farîdûn, sur le mont Damâwend, il sera libéré à la fin des temps, mais il sera alors vaincu par le héros Karasaspa: voir A. CHRISTENSEn, Les Gestes des rois dans les traditions de l'Iran antique, Paris, 1936, p. 17-19 et J. R. Hinnells, «Zoroastrian Saviour Imagery and its Influence on the New Testament», Numen, 16/3 (1969), p. 172.

8. E. D. Van Buren, «The Dragon in Ancient Mesopotamia», Orientalia, 15 (1946), p. 1-45.

9. Ibid.,p. 45. Le serpent était, semble-t-il, un thème distinct du dragon, et c'est lui que nous voyons affronté ou enlacé comme les serpents gémeaux utilisés sur les sceaux babyloniens. Cette représentation dérivait en partie de la croyance selon laquelle le serpent est androgyne, à la fois principe mâle et femelle, voir E. D. VAN BuREN, «Entwined Serpents », Archiv für Orientforschung, 10, (1935-1936), p. 53-54.

10. A. Kalantar, Armenia from the Stone Age to the Middle Ages, Selected Papers, Neuchâtel/ Paris, 1994: «The Stone Age in Armenia», p. 15-28 (et surtout p. 22-24), et «An Ancient Irrigation System», p. 29-35.

11. G. ÖNEY, Anadolu Selçuklu Mimarisinde Süsleme ve el Sanatlari, Architectural Decoration and Minor Arts in Seljukj Anatolia, Ankara, 1978, p. 143 sq.

12. Les croyances des Chinois érigent le Dragon vert de l'Est au rang de divinité cardinale aux côtés du Tigre blanc, de l'Oiseau de feu et de la Tortue entourée d'un serpent. Il revêtait en outre une symbolique cosmologique comme maître du monde souterrain, mais il restait le plus souvent associé à la fertilité et à la pluie, voir J. Rawson, Chinese Ornament, The Lotus and the Dragon, Londres, 1984, p. 90. Par ailleurs, le dragon est l'un des motifs archétypaux de l'art et de la symbolique chinoise. À l'origine, tous les arts seraient nés de la spirale initiale matérialisée sous la forme du dragon, J.-P. Diény, Le Symbolisme du dragon dans la Chine antique, Paris, 1987, p. 3. 
Le dragon était un emblème politique et cosmologique associé dès l'époque Han à l'empereur qui seul revêtait un vêtement particulier à douze emblèmes appelé "gün», et portant comme motifs la Lune, le Soleil et le dragon ${ }^{13}$. Le dragon est également l'un des animaux de l'horoscope chinois qui fut aussi celui des tribus turques avant qu'elles n'adoptent les croyances islamisées d'origine grecque. Le dragon est visible sur une des fresques murales de Kotscho, dans le bassin de Turfân, aux côtés des autres animaux ${ }^{14}$ : rat, chèvre, coq, tigre, etc.

Dans le milieu culturel turc, le dragon est un vieil emblème totémique que possédaient certaines tribus et qui se perpétua sur l'étendard de Tamerlan $\mathrm{au} \mathrm{XIV}^{\mathrm{e}}$ siècle $^{15}$. Il est à la fois un signe cosmologique, totémique et magicoreligieux utilisé en tant que tel par les shamans ${ }^{16}$, et auquel certaines tribus rendaient un culte ${ }^{17}$.

Le dragon des Beatus, un des symboles du Mal, n'est pas sans rappeler son homonyme, le dragon arménien et cilicien, thème fréquent et important dans le registre iconographique des bas-reliefs sculptés des églises et des évangéliaires arméniens, dans la représentation notamment de saint Georges ou saint Théodore terrassant le dragon. D'après Arménag Sakisian ${ }^{18}$, le dragon arménien serait connu dans l'art local dès le viII siècle et se définit comme «un dragon sans pattes ni ailes et son corps est noué au centre ${ }^{19}{ }$, comme cela est visible sur le basrelief de l'église de Akhthamar datant du début du $x^{e}$ siècle $^{20}$, dans les chapiteaux

13. Ibid., p. 189-190.

14. A. von Le CoQ, Die buddhistische Spätantike in Mittelasien, IV, Atlas zu den Wandmalerein, Berlin, 1924, pl. 17.

15. Les dragons étaient nombreux dans l'art scythe et chez les peuples turco-mongols de Sibérie occidentale. Ce dragon tire donc ses origines d'anciens totems sibériens où il était associé au loup, trace lointaine d'une ancienne divinité céleste datant du second millénaire av. J.-C. E. Esın, «Tös and Monçuk, Notes on Turkish Flag Pole Finials», Central Asiatic Journal, 16 (1972), p. 14-36. Voir A. GRÜNwEDEL, Altbuddhistische Kultstätten in Chinesisch-Turkistan, Berlin, 1912, fig. 356, fig. 617 et les études d'E. Esin, Le Dragon dans l'iconographie turque, Paris IV-Sorbonne, doctorat de troisième cycle, 1969, p. 32-53, et ID., Antecedents and Development of Buddhist and Manichean Turkish Art in Eastern Turkestan and Kansu, Istanbul, 1967, p. 55.

16. J.-P. Roux, La Religion des Turcs et des Mongols, Paris, 1984, p. 128.

17. E. Esin, «The Cosmic Symbolism of the Dracontine Arch and Apotropaic Mask in Turkish Symbolism », Art and Archeology Research Papers, 4 (1973), p. 32. Le moment de l'année correspondant à la manifestation du dragon des pluies commençait au solstice d'hiver dans le signe du Capricorne et on le figurait dans l'art comme une arche formée d'un double dragon affronté, fréquemment représentée sur les pierres tombales des Turcs sibériens du $\mathrm{VI}^{\mathrm{e}}$ au $\mathrm{VIII}^{\mathrm{e}}$ siècle, puis sur les tombes saljoukides: ibid., p. 33 et pl. 7 et 8 .

18. A. B. SAKISIAN, «Thèmes et motifs d'enluminures et de décorations arméniennes et musulmanes», Ars Islamica, 6 (1939), p. 66-87 (p. 83).

19. Ibid., p. 84.

20. W. Bachmann, Kirchen und Moschen in Armenien und Kurdestan, Leipzig, 1913, pl. 37. 
de la bibliothèque de Sanahin datant du $\mathrm{XI}^{\mathrm{e}}$ siècle $^{21}$, dans les bois sculptés de la porte du couvent des Apôtres exécutée en $1134^{22}$, dans une miniature d'un évangéliaire arménien réalisé au XIV ${ }^{\mathrm{e}}$ siècle $^{23}$, dans un émail cloisonné géorgien ${ }^{24}$ ou dans le Cifte Minareli d'Erzurum construit et sculpté au XII siècle par des artisans arméniens ${ }^{25}$.

En Arménie et en Géorgie, les saints guerriers tueurs de dragons sont des thèmes décorant fréquemment les bas-reliefs à l'entrée ou sur les façades des églises ${ }^{26}$, comme on peut les voir sur la frise de l'abside ouest de l'église de Martvili datant au plus tôt du vII ${ }^{\mathrm{e}}$ siècle ou, au plus tard, du règne du roi Georges d'Abkhasie au xe siècle (912-957) ${ }^{27}$.

On observe le même thème sur deux édifices: la façade de l'église SainteCroix d'Akhthamar datant du $\mathrm{x}^{\mathrm{e}}$ siècle (921), où saint Théodore, une fois encore, tue un dragon dont le corps est pourvu d'un nœud en forme de cœur ${ }^{28}$, comme sur le tympan ouest de l'église de Nicorzminda en Géorgie (planche 19), datant du XI ${ }^{e}$ siècle et bâtie par le roi Bagrat IV (1027-1072) ${ }^{29}$. Le nœud en forme de cœur est donc un motif présent en Orient un quart de siècle au moins avant son apparition dans les miniatures du premier Beatus, peut-être peint à Tábara, conservé à la Pierpont Morgan Library à New York et datant de 940-945. Dans les deux cas, le dragon est associé au Mal suprême, l'un combattu par un saint, l'autre faisant ses dernières apparitions à la fin des temps.

21. A. B. SAKISIAN, «Thèmes...», p. 83. Au sujet de la communication de O. K. Werckmeister, «Islamische Formen in spanischen Miniaturen des 10. Jahrhunderts und das Problem der mozarabischen Buchmalerei», dans L'Occidente e L'Islam nell'alto medioevo, vol. II, Spolète, 1965, p. 933-968 et p. 978, A. Grabar rappelle à l'auteur l'importance de la Transcaucasie dans l'assimilation des éléments islamiques (Géorgie, Arménie) aux $\mathrm{X}^{\mathrm{e}}$ et $\mathrm{XI}^{\mathrm{e}} \mathrm{s}$.

22. A. B. SAKISIAN, «Thèmes...», p. 83.

23. G. Didi-Huberman éd., Saint Georges et le Dragon, Versions d'une légende, Milan, 1994, p. 92 : Évangiles, Saint Georges et le dragon, XIv ${ }^{\mathrm{e}} \mathrm{s}$., Erevan, Matenadaran, $\mathrm{n}^{\circ} 6305, \mathrm{f}^{\circ} 282 \mathrm{r}^{\circ}$ et voir La Miniature arménienne, XIII ${ }^{e} X I^{e}$ siècle, Collection du Matenadaran, Erevan/Léningrad, 1984 : Évangiles, Saint Georges au dragon, $\mathrm{n}^{\circ} 7699$, Crimée, $\mathrm{XIV}^{\mathrm{e}}$ s., $\mathrm{f}^{\mathrm{0}} 282 \mathrm{r}^{\circ}$.

24. G. Didi-Huberman éd., Saint Georges...,p. 92 : émail cloisonné, Saint Georges et le dragon, Anonyme géorgien, $\mathrm{XV}^{\mathrm{e}} \mathrm{s}$., Tbilissi, musée d'Art géorgien.

25. A. B. SAKISIAN, «Thèmes...», p. 84.

26. S. CARboni a évoqué l'origine du thème dans un chapitre de son Ph. D. (The Wonders of Creation and the Singularities of Ilkhânid Painting, A Study of the London Qazwînî, B. L. Ms. Or. 14.140, Ph. D., SOAS, Londres, 1992 : «The Knotted Dragon», p. 475-479) et il conclut également sur l'origine chrétienne et caucasienne du dragon à nœud en forme de cœur.

27. J. Baltrusattis, Études sur l'art médiéval en Géorgie et en Arménie, Paris, 1929, p. 58 et pl. LXVII, p. 67.

28. J. C. Davies, Medieval Armenian Art and Architecture, The Church of the Holy Cross, Aght'amar, Londres, 1991, pl. 35, les trois saints équestres, 102 et W. Bachmann, Kirchen..., pl. 37.

29. W. Bachmann, Kirchen..., p. 58 et pl. XLVII. 
La fréquence du nœud parant les dragons en Orient, dragons présents dans l'art chrétien mais aussi dans l'art islamique, nous autorise d'ores et déjà à émettre l'hypothèse d'une origine orientale du thème par le biais des liens commerciaux et culturels entre les communautés chrétiennes, celle d'Orient, Arméniens ou Syriaques, et les Mozarabes, sans que le thème initial ne soit obligatoirement issu d'un saint combattant le dragon puisque aucune des miniatures représentant dans les Beatus les saints cavaliers ou saint Michel terrassant le dragon n'est décorée du nœud du dragon.

En Orient, à partir du $\mathrm{IX}^{\mathrm{e}}$ siècle, le nœud acquiert un sens renouvelé lié à l'élaboration d'une véritable doxa par l'astrologue Abû Ma'shar al-Balkhî et son Madkhal ou Grande introduction à l'astrologie ${ }^{30}$. L'image du nœud parant le corps du dragon est désormais assortie d'une signification astrologique et magique dans l'art arabo-musulman médiéval. Cette dernière a été mise en évidence dans un article magistral de Willy Hartner ${ }^{31}$. Aux origines de ce symbole se trouve une légende: un anti-dieu hindou, Râhu, tenta de dérober le soma, breuvage d'immortalité des dieux, mais il fut dénoncé par la Lune et le Soleil et, en guise de punition, il eut la tête tranchée. Poursuivant de sa vengeance les deux luminaires, il tente en permanence de les dévorer. Ainsi se trouve résumée la cause des éclipses soli-lunaires, provoquées par la vindicte du dragon ${ }^{32}$.

Le dragon, désormais placé dans les cieux, devint une pseudo-planète ${ }^{33}$ utilisée par les astrologues afin d'indiquer la position mobile des nœuds lunaires où se produisent les éclipses dont la position change tous les dix ans, la Queue occupant alors la place de la Tête, une révolution complète s'effectuant en dixhuit ans et demi ${ }^{34}$; la Tête, Râhu, fut le nœud ascendant de l'orbite lunaire et Kêtu, sa Queue, le nœud descendant ${ }^{35}$. L'art hindou les représenta aux côtés des autres planètes, la Tête comme une tête monstrueuse et la Queue comme un

30. ABÛ MA'shar, Kitâb al-Madkhal al-Kabîr ilâ 'ilm ahkâm al-nujûm, Liber Introductorii Maioris ad Scientiam Judiciorum Astrorum (éd. R. LemAy, Naples, 1995).

31. W. Hartner, «The Pseudo-Planetary Nodes of The Moon's Orbit in Hindu and Islamic Iconography», Ars Islamica, 5 (1938), p. 113-154. Comme l'explique G. Furlani, les Bayloniens n'imputaient pas l'éclipse à un dragon, mais en revanche le concept apparaît chez les Syriaques via l'astrologie iranienne et ensuite arabe: voir G. FURLANI «Tre trattati astrologici siriaci sulle eclissi solare e lunare», dans Atti della Accademia Nazionale dei Lincei (Classe di Scienze Morali, Storiche e Filologiche), 344 (1947), p. 569-606 (p. 570-586) et G. DE CALLATAŸ, «The Knot of the Heavens », Journal of the Warburg and Courtauld Institutes, 59 (1996), p. 1-13.

32. Bhâgava Purâna, 10, 8, 9, dans A. DaniéLou, Mythes et dieux de l'Inde, Paris, 1992, p. 478479; B. BhatTACHARYA, Iconography of Buddhism, Calcutta, 1958, p. 377-378.

33. E. Sachau, Al-Bîrûnî's India, An Account of the Religion, Philosophy, Literature, Geography, Chronology, Astronomy, Customs, Laws and Astrology of India, 1888, réimpr. Londres, 1910, vol. 2, p. 107-108: description du dragon selon les sources astrologiques hindoues.

34. W. HARTNET, art. «al-Djawzahr », E.I. ${ }^{2}$ II, p. 515.

35. S. SivaPriYAnANDA, Astrology and Religion in Indian Art, New Delhi, 1990, p. 74-77. 
hybride à queue ophidienne. De plus, chacune de ces entités, comme les autres planètes, eut droit à un domicile dans l'un des douze signes du zodiaque, la Tête en Gémeaux et la Queue en Sagittaire ${ }^{36}$.

Ce dragon céleste est également présent dans la cosmologie zoroastrienne dans laquelle il symbolise la voie lactée ${ }^{37}$ et le nom même de cette planète, «al-Jawzahar», d'origine indienne, retranscrit en pehlevi dès le $\mathrm{vI}^{\mathrm{e}}$ siècle av. J.-C., fut ainsi transmis au monde arabo-musulman médiéval ${ }^{38}$.

De façon plus générale, le dragon entourant le cosmos de son corps - ou ouroboros - symbolise dans les diverses mythologies (grecque en particulier) le temps éternel et permanent ${ }^{39}$, thème que les alchimistes reprendront comme celui de la transmutation des métaux en général et de la dissolution des corps par fermentation en particulier ${ }^{40}$, et que les gnostiques utilisèrent dans bon nombre d'amulettes ${ }^{41}$.

Ce dragon ne figura cependant jamais au rang d'une véritable planète puisque al-Jawzahar ne fut que très exceptionnellement représenté et il ne prit jamais place - sauf exception - aux côtés des sept planètes, dans les miniatures comme dans les métaux ${ }^{42}$.

Les astrologues du monde arabo-persan médiéval connaissent certes les origines hindoues des nœuds ${ }^{43}$, et mentionnent les deux signes abritant en

36. A. Bouché-LeClerq, L'Astrologie grecque, Paris, 1899, p. 122-123.

37. J. Duchesne-Guillemin, «L'église sassanide et le mazdéisme», dans C. Puech éd., Histoire des religions, Paris, 1972, p. 16 et D. N. MAcKenzIE, «Zoroastrian Astrology in the Bundahishn», BOAS, 27 (1964), p. 525. A. PANAINo, Tessere il cielo, Considerazioni sulle Tavole astronomiche, gli Oroscopi, et la dottrina dei Legamenti tra Induismo, Zoroastrismo, Manicheismo e Mandeismo, Rome, 1998, p. 71-86, et dans les autres croyances, manichéisme par exemple, p. 93.

38. W. HARTNER, art. «al-Djawzahr», E.I. ${ }^{2}$ II, p. 514.

39. W. Deonna, «Ouroboros », Artibus Asiae, 15, 1/2 (1952), p. 163-170 et L. CharbonneauLassay, Le Bestiaire du Christ, réimpr. E. J. Toth, Milan, 1974, p. 804.

40. Ibid., p. 805-806.

41. Pistis Sophia, Le Livre sacré des gnostiques d'Égypte, trad. F. Bardeau, Paris, 1977, p. 130 ; K. Seligmann, Le Miroir de la magie, Paris, 1989, p. 64-70.

42. En revanche, le dragon ouroboros fut important dans toute la littérature gnostique traduite en arabe comme le traité d'Olympiodore sur la pierre des philosophes, voir E. BLOCHET, «Études sur le gnosticisme musulman», Rivista degli studi orientali, 4 (1911), p. 30.

43. AL-BîRÛNî, Tafhîm, The Book of Instruction in the Elements of the Art of Astrology, Written in Ghaznah on 1029 a. d., Reproduced from British Museum Ms. Or. 8349, éd. R. R. WRIGHT, Londres, 1934, n 177, p. 91 («Mâ al-Jawzahar: ces orbites sont inclinées vers l'écliptique. Il y a deux points opposés à l'intersection : le nœud ascendant, la Tête et le nœud sud, la Queue») et p. 253 ( «Il est dit que les Babyloniens pensaient que le nœud ascendant augmente les effets [bienfaisants ou malfaisants] des planètes»). 
domicile le Dragon: les Gémeaux et le Sagittaire ${ }^{44}$, mais ne donnent jamais d'indications sur son apparence. En fait, même si le Dragon des éclipses de Lune demeura une planète fictive, celle-ci fut représentée par un symbole ou de façon détournée, parfois même par un ou deux dragons, et fit évoluer de façon décisive l'iconographie des deux signes du zodiaque qui l'hébergent en domicile ${ }^{45}$.

Le dragon, qui réapparaît et se multiplie au XIII ${ }^{\mathrm{e}}$ siècle à la période saljoukide et ilkhanide dans les métaux ou les bas-reliefs, est ainsi porteur de multiples significations propres à l'histoire et à l'héritage des tribus turques, mais aussi aux traditions millénaires enracinées dans ce milieu mésopotamien ${ }^{46}$.

\section{Les représentations du Dragon al-Jawzahar, pseudo-planète, dans l'art musulman}

L'arrivée des Turcs saljoukides porteurs d'une culture et d'un art où le dragon était un thème connu, voire habituel, de la symbolique religieuse, cosmologique ou politique, fut le facteur déterminant de la diffusion de cette nouvelle planète ${ }^{47}$.

Par ailleurs, d'autres détails iconographiques, dont les nœuds en forme de cœur que l'on trouve dans le corps de ces dragons et dont l'importance n'est pas des moindres, semblent, eux, avoir une origine propre à la haute Mésopotamie et à l'Est de l'Anatolie.

La plus ancienne représentation d'un dragon à connotation astrologique dans l'art islamique est celle du dragon qui ornait avec les sept autres planètes (chacune accompagnée du signe dans lequel elle est en exaltation) le pont de Jazîra ibn 'Umar sur le Tigre, pont construit par Jalal al-dîn en 559 H/1164. Le dragon est ici associé au Sagittaire dans lequel la Queue est en domicile, et il

44. ABÛ MA'SHAR, The Abbreviation of the Introduction to Astrology together with the Medieval Latin Translation of Adelard of Bath (éd. C. Burnett, K. Yamamoto, M. Yano, Leyde/New York/ Cologne, 1994, p. 16 et 21).

45. A. CAIOzzo, Images du ciel d'Orient au Moyen Âge, Paris, 2003, p. 224.

46. G. Curatola, «The Viar Dragon», Soltaniyya III, Quaderno del Seminario di iranistica uralo-altaistica e caucasologia dell'universita degli studi di Venezia, Venise, 1982, p. 78.

47. Cette théorie centrale est développée par deux historiens de l'art turc: E. Esin, Le Dragon dans l'iconographie turque, et G. ÖNEY dans les articles cités, qui analysent de façon très détaillée tous les monuments porteurs de symboles récemment introduits en Anatolie et en haute Mésopotamie (lion, aigle, "anqa", arbre de vie, etc.) qu'ils attribuent exclusivement à la culture turque marquée par l'Asie centrale et orientale. De leurs arguments essentiellement ethnocentristes, ils évacuent le plus souvent d'autres interprétations, notamment astrologiques, intégrées par G. AzARPAY, «The Eclipse Dragon on an Arabic Frontispice Miniature», JAOS, 98 (1977), p. 363-374. 
figure dans le corps d'un centaure dont la queue s'achève par un dragon à gueule énorme, nez flexible comme une trompe et corps ophidien marqué par un nœud ${ }^{48}$.

Ce dragon des éclipses soli-lunaires possède en général une ou deux caractéristiques iconographiques fondamentales qui permettent de le repérer dans l'art: il est le plus souvent figuré comme deux dragons affrontés ou en visà-vis, et dotés d'un ou plusieurs nœuds en forme de cœur dessinés par le cours sinueux de leurs corps. Le choix de cette iconographie remonte peut-être à l'Asie centrale, puisque sur les fresques murales de Bäzäklik, datant du IX siècle, on peut voir deux dragons enlacés ${ }^{49}$.

Les dragons dressés et le plus souvent affrontés sont présents sur de nombreux supports, pierres, métaux et miniatures.

En 618/1221, le calife abbasside al-Nâsir fit édifier à Bagdad une porte que l'on nomma la porte du Talisman dont la partie supérieure du linteau était décorée d'un bas-relief présentant deux dragons gueule béante, le corps paré d'un nœud en forme de cœur maîtrisé par la langue par un personnage central nimbé ${ }^{50}$. Certains ont voulu y voir la victoire du calife sur les Mongols ${ }^{51}$ et sur le Khwârism Shâh Muhammad II en 617/1220 et 618/122152; pour d'autres, elle a une portée clairement apotropaïque ${ }^{53}$; mais on peut aussi retenir le sens astrologique en raison du personnage central nimbé qui n'est pas sans analogies avec la représentation habituelle et canonique de la Lune.

Ces dragons si particuliers au corps décoré d'un nœud en forme de cœur sont des thèmes assez fréquents dans l'architecture turque d'époque saljoukide ${ }^{54}$,

48. W. Hartner, The Pseudo-Planetary, p. 114 et 120, et J. Gierlichs, Mittelalterliche Tierreliefs in Anatolien und Nordmesopotamien, Untersuchungen zur figürlichen Baudekoration der Seldschuken, Artuqiden und ihrer Nachfolger bis ins 15. Jahrhundert, Tübingen, 1996, p. 250252, pl. 62-63-64.

49. A. von Le CoQ, Facsimile-Wiedergaben der wichtigeren Funde der ersten Königlich preussischen Expedition nach Turfan in Ost-Turkestan. Im Auftrage der Generalverwaltung der Königlichen Museen aus Mitteln des Baessler-Institutes, Berlin, 1913, pl. 32.

50. M. Van Berchem, J. Strzygowski, 'Amida, Matériaux pour l'épigraphie et l'histoire musulmanes du Diyar Bakir, Paris, 1910, p. 83. Voir F. SARRE, E. HerzFeld, Archäologische Reise im Euphrat und Tigris Gebeit, 1, Berlin, 1920, 4 et 2, pl. X et XI.

51. A. Hartmann, An-Nâsir li-Dîn Allâh (1180-1225), Politik, Religion, Kultur in der späten 'Abbasidenzeit, Berlin/New York, 1975, p.162-166, Bâb al-Tîlasm, qui fait un récapitulatif de la polémique concernant la signification de la porte.

52. F. SARRE, E. HeRZFeld, Archäologische ..., vol. I, p. 35-36.

53. E. Kunnel, «Drachensportale», Zeitschrift für Kunstwissenschaft, 4 (1950), p. 1-18.

54. G. ÖNEY, «Dragons Figures in Anatolian Seljuk Art», Belleten, 33 (1969), p. 192-216 et, plus récemment, O. PANCAROǦLu, «The Itinerant Dragon-Slayer: Forging Paths of Image and Identity in Medieval Anatolia», Gesta, 43/2 (2004), p. 151-164. 
comme le montre le bas-relief de la porte d'un caravansérail à Sinjar, construit sous le règne de Badr al-dîn Lû'lû (1219-1259) ${ }^{55}$.

D'autres bas-reliefs à dragons au corps paré de nœuds ornent d'autres portes: celle d'Urfa à Diyâr Bakir, celle de la citadelle de Konya ${ }^{56}$, ou la porte monumentale du Karatay Khân (1240) de la même ville ${ }^{57}$, ou la porte d'Urfa (Édesse) de la citadelle d'Alep en Syrie ${ }^{58}$. Le thème est fréquent dans les décors des palais (celui d'Alâ'al-Dîn à Konya, 1220-123759, celui du Sultan à Kaysare, $\left.1232-1236^{60}\right)$.

Ce thème redondant et répandu serait en fait une sorte de talisman qui permet de garantir le triomphe de la lumière, la fertilité et l'eau en devenant dragon de la pluie, car le dragon turco-chinois dévorant la Lune possédait en outre la propriété d'attirer la pluie et il était considéré comme un symbole de fertilité ${ }^{11}$.

Outre les décors sculptés, les métaux anatoliens, mésopotamiens ou iraniens sont nombreux à le représenter. On connaît de nombreux heurtoirs de bronze décorant les portes des cités sous la forme de deux animaux affrontés ou opposés et enlacés par la queue, parfois séparés par une tête de fauve (peut-être un lion). Ceux de l'Uû Cami de Cizre (al-Jazîra) à Copenhague ${ }^{62}$ et du Dahlem Museum à Berlin ${ }^{63}$ sont fidèles à la description de celui ornant la porte d'Alep de la cité d'Amida faite par al-Jazarî ${ }^{64}$ dans son traité sur les automates et reproduit dans les ouvrages enluminés dès la première copie de $1203^{65}$ :

Puis je fis pour chaque volet (de la porte), une sonnette (un heurtoir) en cuivre moulé sous l'aspect de deux serpents reliés, la tête de l'un face à celle de l'autre. Leurs bouches sont ouvertes et elles veulent dévorer le cou et la tête d'un Lion.

55. F. Sarre, E. Herzfeld, Archäologische..., 1, p. 13-15 et F. SARre, Der Kiosk von Konia, Berlin, 1936, pl. 25.

56. G. InAL, «The Place of the Dragon Relief at Susuz Han in the Asian Cultural Circle», Sanat Tarihi Yigilli, 4 (1970-1971), fig. 1, 2 et 32.

57. Ibid.,p. 198, fig. 10, toujours sous le règne d'Alâ'al-Dîn Kay Qûbadh.

58. A. GABRIEL, Voyages archéologiques dans la Turquie orientale, vol. I, Paris, 1940, p. 166.

59. G. ÖNEY, «Dragons Figures...», p. 196: Konya, Ince Minarelli Medrese Museum, inv. $\mathrm{n}^{\circ} 580$ et F. SARRE, Der Kiosk...

60. Ibid., p. 197, fig. 6 et 7.

61. Ibid., fig. 28, 29 et 32, p. 193-216; J.-P. DiéNY, Le Symbolisme..., p. 195.

62. Ibid., p. 201, fig. 17 .

63. Ibid., p. 201, fig. 18: Berlin, Dahlem Museum, inv. I. 2242.

64. M. Van Berchem, J. Strzygowski, Amida, p. 75, 78-81. Selon Van Berchem, les portes d'Amida étaient ornées de bas-reliefs sculptés dont l'aigle ou la chouette, emblème de l'artouqide Muhammad, mais aussi d'un aigle agrippant une tête de Taureau, symbole de la victoire des Artouqides sur les Inâlides.

65. AL-JAZARî, The Book of Knowledge of Ingenious Mechanical Devices (éd. D. Hitl, Boston/ Dordrecht, 1974, p. 191 et 194, fig. 148). 
Nous pouvons voir dans toutes les copies une représentation similaire: deux serpents aux allures de dragons, dressés, lovés sur eux-mêmes, et aux queues entrelacées. Gueule béante, ils paraissent s'affronter. Entre les deux gueules se trouve une tête de fauve retenue par les langues des dragons qui lui servent de support. La signification de ce heurtoir pourrait être astrologique. En effet, hormis le zodiaque de l'horloge à eau, qui est un zodiaque des signes d'après al-Jazarî, certains personnages et certains symboles semblent relever de la représentation des planètes telles que nous les voyons dans les autres manuscrits ${ }^{66}$. Les deux dragons affrontés, comme ceux de la porte du Talisman ou de Susuz Khân, attaquent cette fois une tête de lion, lequel est d'un point de vue astrologique le domicile du Soleil, menacé (comme la Lune) par le Dragon. Le heurtoir, placé à l'entrée de la ville, jouerait de ce fait le même rôle que la porte du Talisman en écartant certaines calamités, dont les éclipses.

Les monnaies artouqides présentent parfois des dragons enlacés, en particulier sur celles de Qara Arslân, chef de Diyâr Bakir (1109-1144) ${ }^{67}$. Dans le Vaso Vescovali, qui présentait dès le début du XII siècle les planètes telles que nous les voyons dans le manuscrit de la BnF Sup. pers. 174, la huitième planète est représentée aux côtés des sept autres ${ }^{68}$. Elle apparaît certes dans des domiciles: Râhu, «al-Ra's» logé dans les Gémeaux et Kêtu, Dhanab al-Tinnîn dans la queue du Sagittaire. La planète al-Jawzahar, elle, figure dans une rondelle sous la forme d'un prince encadré par deux hampes à tête de dragon aux pieds desquelles se tiennent deux courtisans ou serviteurs. Ce type de représentation est assez courant dans les bols et chaudrons du Khorasân, en particulier dans une boîte circulaire du XII ${ }^{\mathrm{e}}$ ou du XIII ${ }^{\mathrm{e}}$ siècle (ex-Minassian Collection) décorée de signes zodiacaux. Au centre, une représentation quasi identique entourée sur le bord inférieur par une frise de sphinx.

Par ailleurs, de nombreux métaux nous montrent un personnage assis sur un trône et serrant dans ses mains les bras du siège représentant des têtes de dragons. Cette représentation s'apparenterait à celle d'un luminaire (en l'occurrence, le Soleil) terrassant le dragon des éclipses comme le pense E. Baer ${ }^{69}$.

66. C'est le cas des femmes musiciennes (Vénus), du guerrier vêtu de rouge tenant une épée (Mars), du cornac indien (Saturne), du scribe (Mercure), etc.

67. S. Lane-Poole, Coins of the Turkumân, Seljuk, Urtuk, Zengee Houses in the British Museum, Cl. X-XIV, Londres, III, 1877, pl. 7.

68. Miniature $\mathrm{f}^{\circ} 83 \mathrm{r}^{\circ}$ dans M. BarruCAND, «The Miniatures of the Daqâ' $i q$ al-Haqấ'iq (B.N. persan 174): A Testimony to the Cultural Diversity of Medieval Anatolia», Islamic Art, 4 (1991), pl. C.

69. E. BAER, «The Ruler in Cosmic Setting: A Note on Medieval Islamic Iconography », dans A. Daneshvari éd., Essays on Islamic Art and Architecture, In Honor K. Otto-Dorn, Malibu, 1981, p. 13-19. 
L'âge d'or des dragons affrontés, après ceux de la période saljoukide, fut sans conteste l'art timouride ${ }^{70}$. De nombreux objets portent des dragons en vis-à-vis : la garde en ivoire d'une épée ${ }^{71}$, un presse-papiers ${ }^{72}$, des chandeliers ${ }^{73}$ aux têtes de dragons en vis-à-vis sont les plus symboliques, sans compter les tasses en jade ${ }^{74}$ et les jarres en métal ${ }^{75}$ à anses de dragons, les coffres et objets en bois sculpté décorés du même motif ${ }^{76}$, dont beaucoup ont appartenu au prince Ulugh Beg, féru d'astronomie et astrologue de talent qui ne pouvait ignorer la signification de pareilles représentations.

La plus ancienne apparition du Dragon de l'écliptique dans l'art du livre appartient au Livre des antidotes (Paris, BnF, ms. ar. 2964) daté de 1199 (planche $22)^{77}$. Le double dragon encercle la Lune, comme s'il l'avait en quelque sorte avalée, mais la Lune présentée en souveraine brandit le croissant fermé devant sa tête et les anges semblent protéger la scène qui peut ainsi se résumer, comme dans la porte du Talisman, en une représentation apotropaïque. D'après Guitty Azarpay, la forme circulaire de ce dragon des éclipses serait une réminiscence de l'ouroboros connu des Babyloniens, comme le montrent certains sceaux de l'époque sassanide ${ }^{78}$ et comme l'évoque encore le Bundahishn ${ }^{79}$.

70. E. J. Grube, «Notes on the Decorative Arts of the Timurid Period, II», Islamic Art, 3 (1989), p. 175-208.

71. Sabre à poignée en forme de dragons en vis-à-vis, Istanbul, Topkapi Sarayi Armory, inv. I-220, Iran ou Asie centrale, XV s. $77,5 \mathrm{~cm} ; 10,5 \mathrm{~cm}$, dans T. Lentz, G. D. Lowry, Timur and the Princely Vision, Persian Art and Culture in the Fifteenth Century, Washington, 1989, p. 222.

72. Presse-papiers en jade, New York, Metropolitan Museum of Art, inv. 1902, 02.18.765, Iran ou Asie centrale, 1400-1450, dans T. LenTZ, G. D. Lowry, Timur ..., p. 143.

73. Chandelier, Copenhague, The David Collection, inv. 38/1982, Iran ou Asie centrale, $\mathrm{xv}^{\mathrm{e}}$ s., 25,1 cm; 16,9 cm dans T. LentZ, G. D. Lowry, Timur..., p. 223.

74. Tasse en jade, Londres, San Francisco, Asian Art Museum, The Avery Brundage Collection, B $60 \mathrm{~J} 160$, Iran ou Asie centrale, Xv s., 15,9 cm; 16,5 cm; 12,3 cm, T. LentZ, G. D. Lowry, Timur..., p. 222.

75. Jarre en métal, Londres, the Board of Trustees of the Victoria and Albert Museum, inv. 9431886, Herat, 866H./1461-2, fabriquée par Habibullah Ibn 'Alî Baharjanî, gravée d'argent, 13 cm; 12,8 cm, dans T. Lentz, G. D. Lowry, Timur..., p. 206.

76. Couvercle du coffre d'Ulugh Beg, Istanbul, Topkapi Sarayi Treasury, inv. 2/1846, Asie centrale, 1420-1449, bois gravé avec de la marqueterie polychrome, dans T. LENTZ, G. D. LowRY, Timur..., p. 207.

77. G. Azarpay, «The Eclipse...», p. 363-374 et O. PancaroǦlu, «Socializing Medecine: Illustrations of the Kitāb al-diryāq», Muqarnas, 18 (2001), p. 165-167 et voir l'édition du facsimilé du Livre de la Thériaque, A. CAIOzzo, «Les trois états de la Lune, éléments d'analyse pour la lecture du frontispice du Livre de la Thériaque», dans Commentaires du fac-similé de la Thériaque de Paris, Aboca Museum, 2009, p. 36-47.

78. G. AzARPay, «The Eclipse...», p. 369-371, fig. 6, fig. 8, fig. 9.

79. Textes cosmogoniques d'époque sassanide. $C f$. D. N. MacKenzie, «Zoroastrian Astrology ...», p. 515-516. 
En dépit de la date incertaine de cette miniature, on peut observer dans le manuscrit Paris, BnF Sup. pers. 174, l'ange Shamhûrash, l'équivalent gnostique de saint Georges tuant le dragon ${ }^{80}$, ce qui montre par ailleurs un lien étroit avec le milieu culturel est-anatolien présaljoukide (planche 23).

Par ailleurs, le dragon, à l'image de son homologue chinois, semble revêtir, dans certaines miniatures, une symbolique du pouvoir. Deux dragons chinois en vis-à-vis ornent les trônes du Sultân Luhrâsp, du roi Tahmûras ou du roi Jamshîd dans les miniatures de l'Histoire universelle de Rashîd al-dîn (Bibliothèque de l'université d'Édimbourg, 1306) ${ }^{81}$.

Quant aux nœuds en cœur décorant le corps de la plupart de ces dragons dans les sculptures et les miniatures, ils semblent de toute éternité associés aux représentations des reptiles, à l'instar de la glyptique mésopotamienne datant de près de 2000 ans av. J.-C. ${ }^{82}$. La symbolique du nœud renvoie à celle des dieux lieurs, divinités néfastes et redoutées ${ }^{83}$. Le nœud est d'un usage double: il provoque le mal mais on l'utilise également pour le conjurer ${ }^{84}$.

De ce fait, l'usage de ces nœuds ornant les corps des dragons arméniens et saljoukides n'est sans doute pas anodin. En effet, concepts hérités de l'astrologie hindoue, ils symbolisent désormais les nœuds de Lune ou position des éclipses soli-lunaires ${ }^{85}$, mais en se doublant d'une signification apotropaïque et magique.

De façon isolée, le nœud est à la fois un signe magique et un symbole apotropaïque, et l'on observe une forme similaire dans les alphabets magiques arabes tel le rûhânî, une forme supérieure de magie recommandée par al-Bûnî: f5.Il s'agit ici de l'un des sept signes magiques (parfois après le pentacle,

80. E. Blochet, Études..., 2, 1909, p. 720-726. Sur cet ange et ce dragon, voir A. CAiozzo, «Anges gardiens et génies familiers dans les manuscrits enluminés de l'Orient médiéval», dans J.-P. Boudet, P. Faure, C. Renoux éd., Anges gardiens et démons familiers de Socrate à Tintin, Colloque de l'université d'Orléans, Orléans, 9-10 juin 2006 (Rennes, 2011, p. 97-110).

81. D. Talbot-Rice, B. Gray, The Illustrations of the World History of Rashîd al-Dîn, Édimbourg, 1976, pl. 4, pl. 5, pl. 16.

82. P. Амiet, La Glyptique mésopotamienne archä̈que, Paris, 1980, sceaux n 1247a, 1247b, 1248 , et de façon isolée le thème apparaît dans les sceaux n ${ }^{\circ} 1251$ et 1253, pl. 95.

83. Certaines divinités utilisent le lasso ou le lacet pour neutraliser leurs adversaires, usant ainsi du pouvoir apotropaïque du nœud: en Iran le héros Rustam use de son lasso dans le Shâh Nâmeh de Firdawsî, et avant lui le héros Farîdûn qui lie le tyran Dhâhhâk après sa capture. Cet usage se retrouve dans les mythes sumériens avec Ea et Mardouk liant leurs ennemis. Voir M. EliADE, Images et symboles. Essai sur le symbolisme magico-religieux, le symbolisme du centre, Paris, 1952, p. 132144, et M. EliAde, «Le dieu lieur et le symbolisme des nœuds », Revue de l'Histoire des religions, 67 (1948), p. 5-36.

84. M. Eliade, Images et symboles..., p. 145-146.

85. W. HARTNER, The Pseudo-Planetary..., p. 113-154. 
parfois en dernière lettre), qui signifierait d'après al-Bûnî l'alpha et l'oméga ${ }^{86}$. Ce signe, comme les six autres, est invoqué notamment dans les formules de guérison inscrites sur les bols magiques dans lesquels sont confectionnées les thériaques visant à soigner les piqûres de scorpions et les morsures de serpents ${ }^{87}$. On le retrouve en outre dans divers sceaux et talismans musulmans; signalons à ce propos que l'un des talismans le représentant possède une inscription coranique faisant directement allusion à la ligature des nœuds (Coran CXIII) ${ }^{88}$ :

Je me réfugie auprès du seigneur de l'aube,

contre le mal qu'il créa, contre le mal d'une obscurité quand elle s'étend, contre le mal de celles qui soufflent sur les nœuds et contre le mal d'un envieux qui envie.

Les entrelacs d'époque médiévale formés de nœuds en forme de cœur décorent également les monuments arméniens et géorgiens, notamment à Nicorzminda en Géorgie ${ }^{89}$. On peut noter qu'il s'agit d'un type d'entrelacs formé de deux tiges enlacées ou de deux tiges croisées ${ }^{90}$. Accompagné ou non par des serpents ou des dragons, il devient, de façon isolée, une sorte de motif décoratif dans l'art islamique, présent dans les céramiques mésopotamiennes abbassides dès le $\mathrm{IX}^{\mathrm{e}}$ siècle $^{91}$, puis de façon beaucoup plus généralisée au XIV siècle $^{92}$ à l'image de cette coupe de Raqqa ${ }^{93}$ datant du IX $^{\mathrm{e}}$ siècle selon Marcel Pézard, du XII ${ }^{\mathrm{e}}$ ou $\mathrm{XIII}^{\mathrm{e}}$ siècle selon Ernst Grube ${ }^{94}$. Les métaux sont eux aussi fréquemment décorés

86. E. Doutté, Magie et religion dans l'Afrique du Nord, Paris, 1908, p. 154-156; J. M. G. Dawkins, «The Seals of Salomon», Journal of the Royal Asiatic Society, 1944, p. 150; M. CANova, «Alphabets magiques arabes », Journal asiatique, 1921, p. 37-55 et 1922, p. 250-262 et 259; T. CanaAn, «The Decipherment of Arabic Talisman», Berytus, 4 (1937), p. 103 et Berytus, 5 (1938), p. 143.

87. H. H. SPOER, «Arabic Magic Medicinal Bowls», Journal of the American Oriental Society, 55 (1935), p. 240-244.

88. L. Kalus, Catalogue des cachets, bulles et talismans islamiques, Paris, BnF, n ${ }^{\circ} 13-1$, p. 80.

89. J. Baltrusaitis, Études..., p. 22-23, et pl. XL, nº 62-63-64.

90. Ibid., 2-3, fig. 1 et 2.

91. M. S. Dimand, A Handbook of Muhammadan Art, New York, 1958, p. 367, plat en terre à décor lustré, décor en relief couvert par un vernis jaune avec des taches vertes.

92. J. Sauvaget, Poteries syro-mésopotamiennes du XIV siècle, 1, Documents d'Études Orientales, Institut Français de Damas, Paris, 1932, pl. 16, nº 61 et 63, pl. 18, n 64 et pl. 24, no 93.

93. M. PÉZARD, La Céramique archaïque de l'Islam et ses origines, Paris, 1920, 2, pl. CXLIX.

94. E. J. Grube, «Raqqa Keramik in der Sammlung des Metropolitan Museum in New York», Kunst des Orients, IV (1963), p. 42-78, pl. 12: New York, Metropolitan Museum of Art, plat en céramique, inv. 3471, Fletcher Fund 1934, Raqqa, XII ${ }^{\mathrm{e}}$-XIII ${ }^{\mathrm{e}} \mathrm{s}$., ht.: 7,6 cm; diam.: 26,1 cm, et pl. 15, ACC. N $^{\circ}$ 20-52-3, Rogers Fund, 1920, XI $^{\mathrm{e}}$-XII ${ }^{\mathrm{e}}$ s., même appréciation de la date chez A. Lane, Early Islamic Pottery, Londres, 1947, fig. 81 et 44-45, et chez M. S. Dimand, A Handbook..., fig. 124, p. 193. 
de nœuds, telle la Wade Cup, datée du début du XIII ${ }^{\mathrm{e}}$ siècle, qui comporte, en plus des signes du zodiaque, des nœuds en forme de cœur dont le caractère magique a été souligné par Richard Ettinghausen ${ }^{95}$. Ces derniers s'ajoutent aux poissons et aux sphinx pour renforcer l'aspect astrologique de la coupe qui est sans conteste un objet à forte connotation solaire ${ }^{96}$.

Hormis les nœuds, les têtes de dragons ont souvent pour vocation de rappeler l'existence de cette huitième planète responsable des éclipses, à l'image de celle ornant la queue du Sagittaire et symbolisant la queue du Dragon ${ }^{97}$.

Dans une coupe magique du Sud de l'Anatolie datant du XIII ${ }^{e}$ siècle, les motifs talismaniques lions, scorpions et étoiles à cinq branches sont présents aux côtés de deux dragons affrontés et aux corps noués en forme de cœur ${ }^{98}$. Dans la coupe magique syrienne du XIV ${ }^{e}$ siècle conservée au Louvre, on retrouve cette fois le symbole du nœud sans le dragon. En effet, l'extérieur porte douze médaillons montrant les signes du zodiaque, mais à l'intérieur, entre plusieurs formules de bonne santé, on peut voir d'étranges créatures habituellement utilisées dans les pratiques magiques, talismaniques ou alchimiques: un scorpion, un nœud en forme de cœur, une sorte de lézard ou de salamandre et diverses figures étranges, voire hermétiques. Les nœuds peuvent accompagner les dragons, voire les remplacer à l'occasion par une simple représentation comme dans la coupe magique du Louvre.

Dans les miniatures d'un grand nombre de manuscrits des cosmographies de Qazwînî en langue arabe, à partir du XIV ${ }^{\mathrm{e}}$ siècle, le nœud devient un motif décorant les queues de certains animaux (lions, buffles, chevaux, hybrides). Le Sarre Qazwîn̂̀ en particulier, comme l'a montré Julie Badiee ${ }^{99}$, diffuse une iconographie particulière des planètes et du zodiaque, dérivant certes du Codex Arab. 464, datant de 1280, le plus ancien manuscrit illustré, mais s'individualisant également par des éléments stylistiques et culturels typiquement anatoliens. Ces derniers, tel le nœud lunaire, se retrouvent dans les copies ottomanes ou de l'école du Deccan aux XVI ${ }^{e}, \mathrm{XVII}^{\mathrm{e}}$ et XVIII ${ }^{\mathrm{e}}$ siècles. Stefano Carboni a également

95. W. HARTNER, «Zur astrologischen Symbolik des Wade Cup», Aus der Welt der islamischen Kunst, Festschrift für Ernst Künhel, Berlin, 1959, p. 235-243, et R. EtTinghausen, «The Wade Cup in the Cleveland Museum of Art, Its Origine and Decorations », Ars Orientalis, 2 (1957), p. 327 366.

96. R. Ettinghausen, The Wade Cup..., p. 360.

97. J. GierLich, Drache, Phönix, Doppeladler, Fabelwesen in der islamischen Kunst, Berlin, 1993, p. 40-47.

98. Ibid., 41, nº 8: Coupe magique, Berlin, musée d'Art islamique, inv. I 1992-7, Sud de l'Anatolie, XIII ${ }^{\mathrm{e}} \mathrm{s}$.

99. J. Badiee, An Islamic Cosmography, The Illustrations of The Sarre Qazwîn̂, Ph. D., University of Michigan, 1978, p. 253-255, conclusion p. 345 et tableau p. 339. 
noté la présence de tels motifs symboliques dans le manuscrit B.L. Or. 14.140 qu'il date du début du XIV ${ }^{\mathrm{e}}$ siècle (1305-1315) ${ }^{100}$.

Faut-il conclure à une certaine spécificité des cosmographies en arabe, dans lesquelles le nœud ne conserve plus qu'un lien lointain, voire oublié, avec les phénomènes lunaires? En effet, dans l'ensemble des copies «arabes», le signe des Gémeaux est le plus souvent conventionnel et astronomique (deux jeunes gens enlacés) et le Sagittaire, quoique affublé d'une queue nouée achevée par une tête de dragon, ne tire pas vers elle. En fait, parées de ce nœud, ces constellations ne porteraient-elles pas en réalité la marque discrète d'un autre zodiaque connu à l'époque, le zodiaque astrologique? En effet, les animaux dans lesquels il est présent ne sont pas anodins : il s'agit des dragons, des taureaux et des centaures.

Les dragons ${ }^{101}$ font référence de façon implicite au Dragon des nœuds de Lune: en effet, le Taureau est en astrologie le signe d'exaltation de la Lune, qui se dote de ce nœud, peut-être de façon apotropaïque, afin d'écarter l'obscurité de la Lune; enfin, le nœud est présent dans la queue du Sagittaire, signe de domicile de la Queue du Dragon, ou dans la queue du Centaure ${ }^{102}$ qui, lui, rappelle le Sagittaire. Il serait difficile de n'y voir qu'une série de coïncidences.

Emblème apotropaïque du Proche-Orient ancien, totem des tribus turques, mais incarnant aussi le Mal, le dragon dévore les luminaires, symbolisé par un nœud célébré à titre propitiatoire par l'art musulman ${ }^{103}$.

\section{De l'Orient à l'Occident, migration ou permanence d'un symbole et d'un thème décoratif?}

La présence du nœud dans l'art occidental serait sans conteste, d'après Jurjis Baltrusaitis, une importation orientale en provenance de la Syrie et de la haute Mésopotamie qui produisit bon nombre de supports transportables, tissus, céramiques ou manuscrits ${ }^{104}$. Le manuscrit 644 de la Pierpont Morgan Library fut peint par un moine d'Escala, ville qui accueillait les réfugiés mozarabes

100. S. Carboni, The Wonders of Creation..., 1, p. 475-479 et datation p. 523 sq.

101. J. BADIEE, An Islamic Cosmography, pl. 10, pl. 11, pl. 12, pl. 13.

102. Ibid., fig. 8 .

103. Selon A. B. SAKISIAN, «Les tapis à dragons et leur origine arménienne», Syria, 9 (1928), p. 256, il est difficile d'établir si le thème du dragon trouve son origine dans la communauté arménienne, ou s'il est une importation propre aux Saljoukides. Il est toutefois probable qu'il y eût collusion des deux faits, et que le dragon endogène au milieu syro-mésopotamien, combattu par les saints tueurs de dragons, s'enrichisse sans doute d'une iconographie rénovée sous l'influence des Turcs et devienne de plus porteur du nœud, symbole propre à l'astrologie arabo-persane influencée par l'art et à la symbolique hindoue.

104. J. Baltrusaitis, Le Moyen Âge fantastique, antiquités et exotismes dans l'art gothique, Paris, 1993, p. 93-94, pl. 55, et p. 111-113, pl. 70. 
venant de Cordoue et il aurait pu avoir eu connaissance d'objets d'art décorés de dragons porteurs du nœud en forme de cœur ${ }^{105}$.

Le dragon et surtout son nœud ne seraient d'ailleurs pas la seule importation orientale dans l'art mozarabe, à l'instar du bestiaire fantastique des Beatus, ou d'autres éléments tant décoratifs que thématiques ${ }^{106}$. Ces influences orientales, parfois controversées ${ }^{107}$, ne sont cependant plus à démontrer ${ }^{108}$, surtout concernant les animaux fantastiques ${ }^{109}$. Les thèmes orientaux auraient été copiés à partir de tissus brodés importés de Syrie ${ }^{110}$, ou d'après des manuscrits enluminés ${ }^{111}$ appartenant entre autres aux chrétiens d'Orient ${ }^{112}$; ces derniers étaient assez bien tolérés en Espagne omeyyade ${ }^{113}$ et ils purent y introduire leurs évangéliaires, excellents véhicules iconographiques, porteurs de thèmes

105. J. Williams, The Illustrated Beatus, A Corpus of the Illustrations of the Commentary of the Apocalypse, vol. II, Londres, 1994, p. 27.

106. Ibid., vol. I, p. 143-157.

107. Selon A. GraBAR, dans «Les illuminations des Beatus mozarabes et les miniatures orientales chrétiennes», Cahiers archéologiques, 28 (1979), p. 7, et J. WiLliams, The Illustrated..., vol. I, p. 144 et 151-155. D'après W. Neuss (Die katalanische Bibelillustration..., p. 146-148), l'aspect oriental des Beatus des $\mathrm{X}^{\mathrm{e}}$ et $\mathrm{XI}^{\mathrm{e}} \mathrm{s}$. ne serait dû qu'à leur aspect plat et, d'après J. Williams, ils seraient davantage méditerranéens que proche-orientaux.

108. M. ChURRUCA, Influjo oriental en los temas iconographicos de la miniatura espanola, siglo x al XII, Madrid, 1939, p. 38-71 ; O. K. WeRCKMEISTER, «The Islamic Rider in the Beatus of Girona», Gesta, 36/2 (1997), p. 101-106.

109. J. YARZA LuACES, «Los seres fantasticos en la miniatura castellano-leonesa de los siglos XI y XII», Goya, 103 (1971), p. 7-16 et J. YARZA LuACES, Formas artísticas de lo imaginario, Barcelone, 1987, p. 169-173.

110. A. Grabar signale que, dès le VII s., les chrétiens du Levant transmettent des éléments stylistiques orientaux: «Éléments et islamiques dans les enluminures des manuscrits espagnols du haut Moyen Âge», Arte del Primo Millenio, Atti del II Convegno per lo studio dell'arte dell'alto Medio Evo tenuto presso l'Universita di Pavia nel settembre 1950, Pavie, 1950, p. 312-319 (315) et C. DüBLER, «Las influencias iránicas en la peninsula ibérica», Sefarad, 5 (1975), p. 83-130.

111. R. Wittкower, L'Orient fabuleux, Paris, 1991, p. 88, n 81. R. Wittkower, dans le chapitre «Monstres et merveilles de l'Orient», p. 21-100, note à ce propos, p. 41, que les thèmes de dragons enlacés parvinrent probablement dans les manuscrits occidentaux des XIII ${ }^{\mathrm{e}}$ et XIV ${ }^{\mathrm{e}} \mathrm{s}$. par le biais des cosmographies de Qazwînî. L'auteur souligne plus particulièrement le rôle de Byzance jusqu'au $\mathrm{XII}^{\mathrm{e}} \mathrm{s}$. dans l'introduction d'un bestiaire fantastique en Occident; tout comme C. E. DüBLER, dans «Las influencias iránicas en la peninsula ibérica», Sefarad, 1/V (1945), p. 83-130 et p. 125-127: les alliances et mariages princiers étaient d'excellentes occasions pour se transmettre des présents de toutes natures dont les tissus, objets d'art et manuscrits, comme ce fut le cas lors du mariage de l'empereur Otton II avec la princesse byzantine Théophano.

112. A. Grabar, Les Illuminations..., p. 7-16. A. Grabar note la proximité de styles au sujet du costume de deux moines représentés dans le Beatus de Girona (975), $\mathrm{f}^{\circ} 164 \mathrm{r}^{\circ}$, copiés, sinon inspirés, d'un évangéliaire syriaque montrant saint Éphrem.

113. A. Grabar, Les Illuminations...,p. 8 : «Les Chrétiens du Levant pénétraient sans difficulté dans les provinces d'Espagne conquises par les mêmes Arabes mâtres de la Syrie et de l'Égypte»; H. Terrasse, Islam d'Espagne, une rencontre de l'Orient et de l'Occident, Paris, 1958, p. 40. 
nouveaux préislamiques, parfois marqués par l'art musulman et dont l'art roman et hispanique hérita dès le $\mathrm{x}^{\mathrm{e}}$ siècle ${ }^{114}$ : griffons cornus ${ }^{115}$, sirènes ${ }^{116}$, lions ailés ${ }^{117}$ et rinceaux s'achevant sur des têtes de dragons ${ }^{118}$. À ces thèmes vint donc s'ajouter celui du dragon, présent également sur d'autres moyens de diffusion aisés: les tissus et surtout les tapis arméniens fabriqués en Anatolie par des artisans de Konya et Siwas ${ }^{119}$. Par ailleurs, Lucien-Jean Bord et Piotr Skubiszewski ont bien montré comment le dragon jouait, dans le cas de la Babylone aux serpents, un rôle apotropaïque issu en grande partie de la tradition babylonienne relative aux serpents-dragons ${ }^{120}$.

C'est sans doute par ces voies diverses ${ }^{121}$ que le dragon de l'Apocalypse, symbole du Mal dans tous ses états ${ }^{122}$ (menaçant la Femme vêtue de Soleil, celui de l'adoration de la Bête, celui vomissant des grenouilles et ceux de la Babylone aux serpents), s'enrichit d'un motif de nature cosmologique et astrologique qui vint s'ajouter aux roues à svastika, symboles solaires ${ }^{123}$, fréquentes en Orient et dans l'art omeyyade, visibles en particulier dans les théophanies de l'agneau et devant les pieds des évangélistes, figurant les roues du char céleste de la vision d'Ézéchiel ${ }^{124}$, et à la représentation des cieux en cercles concentriques selon la conception d'Ibn 'Arabî 125 .

Conjuguant les motifs astrologique et magique au thème eschatologique des miniatures, le Dragon des éclipses soli-lunaires offre de ce fait une nouvelle lecture des miniatures illustrant les Commentaires de l'Apocalypse du moine

114. Sur l'ensemble des influences zoomorphes d'origine islamique dans l'art mozarabe, J. G. BECKWITH, «Islamic Influence on Beatus Apocalypse Manuscripts», Acta del simposio para el estudio de los codices del commentario al apocalipsis de Beato de Liébana, Madrid, 1978, I, p. 55-60 et p. 59, et III, pl. 30-33. Sur ces influences dans l'art arménien, S. Der NERSESSIAN, Manuscrits arméniens illustrés des XII ${ }^{e}$ XIII ${ }^{e}$ XIV siècles de la Bibliothèque des Pères Mékhitaristes de Venise, Paris, 1936, p. 23, 49, p. 107-117.

115. S. Der NerSessian, Manuscrits..., p. 23.

116. Ibid., p. 107 et p. 117.

117. Ibid., p. 117.

118. Ibid., p. 23.

119. A. B. SAKISIAN, Les Tapis..., p. 238-256.

120. L.-J. Bord, P. SkUBiszewski, L'Image de la Babylone aux serpents dans les Beatus, Contributions à l'étude des influences du Proche-Orient antique dans l'art du haut Moyen Âge, Paris, 2000, p. 49-80.

121. Ibid., p. 112-117.

122. R. K. EMERson, Antichrist in the Middle Ages, Seattle, 1981, p. 22-113.

123. J. BaLtrusaitis, «Quelques survivances de symboles solaires dans l'art du Moyen Âge», Gazette des Beaux-Arts, 17 (1937), p. 75-82.

124. M. MinTré, La Peinture mozarabe..., pl. 127 : Madrid, B.N., Vit. 14. 2, fo $116 v^{\circ}$, pl. 128 : Beatus de San Millán, Madrid, R. A. H., 33, fo 92r ${ }^{\circ}$ et L.-J. Bord, P. Skubiszewski, L’Image ..., p. 121.

125. М. Churruca, Influjo..., p. 45. 
Beatus, renforçant peut-être la présence du Mal identifié sous la forme de l'Islam qui apporterait les Ténèbres sur le monde.

Mais, en se rapportant cette fois au texte et dans la tradition des cosmologies d'Orient où puisent les récits de l'Apocalypse ${ }^{126}$, le dragon conserverait plutôt ici son antique fonction d'ouroboros ceignant le firmament, ses sept têtes comme autant de planètes, la femme vêtue de Soleil, couronnée de douze étoiles, les signes du zodiaque indiquant les Fixes, trônant sur la Lune, symboliserait la lumière émanant des deux luminaires. Quant au nœud - motif fortuit ou concept déjà connu dans les milieux initiés -, il serait ainsi la huitième planète, le Dragon menaçant perpétuellement la Lumière d'être dévorée lors des éclipses de Soleil et de Lune ${ }^{127}$... Signalons que la Lune fut, en Mésopotamie, un dieu lieur qui luttait contre les Sept, des démons envoyés par l'infernal Nergal, son jumeau cosmique symbolisé par un dragon, afin de la dévorer et de provoquer l'éclipse, sept comme les têtes du dragon de l'Apocalypse ${ }^{128} \ldots$

Paradoxalement, si dans les mythes cosmogoniques du Proche-Orient ancien, le combat cosmique entre le Dragon aquatique (Tiamat) et le Soleil donne naissance au monde et à son humanité ${ }^{129}$, dans l'eschatologie chrétienne du Beatus, dans une situation inversée cette fois, le Dragon, en Léviathan qui ne se nomme pas, accompagné de la Bête surgie de la mer, sont vaincus par les armées du ciel et enchaîné pour être neutralisé à jamais afin que la vie éternelle soit rétablie au seul profit des Justes ${ }^{130}$.

Si la présence du nœud nous interpelle, lui attribuer une signification clairement astrologique est un peu problématique en raison de notre méconnaissance de la transmission des savoirs à cette époque. Le moine enlumineur du premier Beatus illustré l'a-t-il peint de façon fortuite en reproduisant un modèle sans doute oriental, en identifiant le symbole astrologique et magique et ses implications, ou simplement en usant d'un vieux symbole apotropaïque, le nœud?

126. J. DAY, God's Conflict with the Dragon and the Sea, Echoes of a Canaanite Myth in the Old Testament, Cambridge, 1985; M. Fishbane, Biblical Myth and Rabbinic Mythmaking, Oxford, 2003, «The Primordial Serpent and the Secret of Creation», p. 273-292.

127. D. Juste, Les Alchandreana primitifs, Études sur les plus anciens traités astrologiques latins d'origine arabe (X $X^{e}$ siècle), Leyde, 2007, p. 129-135; comme le souligne David Juste, le concept du dragon des éclipses soli-lunaires et ses propriétés astrologiques étaient alors bien présents, connus, et le dragon «représenté», dans les traités astronomiques latins du $\mathrm{x}^{\mathrm{e}}$ s., quoique non utilisé en astrologie pratique (p. 129-130).

128. E. Dhorme, La Religion de Babylonie et d'Assyrie, Paris, 1949, p. 58-59.

129. J. Day, God's Conflict...; L.-J. Bord, P. SkUBISZEWSKi, L'Image ..., p. 112.

130. Sur le Léviathan, le monstre ophidien de l'abîme, voir B. TeYssèdre, Naissance du Diable, De Babylone aux grottes de la mer Morte, Paris, 1985, p. 75-82, et Le Diable et l'Enfer au temps de Jésus, Paris, 1985, p. 152-187. 
Mais, par-delà les mythes et les religions, accidentelle ou intentionnelle, l'alliance entre nœud et dragon témoigne aujourd'hui encore des terreurs millénaristes, peurs que certains de nos contemporains ont encore manifestées en l'été 2000 en percevant l'éclipse comme annonciatrice de l'Apocalypse...

Anna CaIozzo - Université Paris 7 Denis Diderot, EA 337 Identités Cultures Territoires, case courrier 7001, 5 rue Thomas Mann, 75253 Paris Cedex 13

Éclipse ou Apocalypse, remarques autour du nœud du dragon dans les miniatures des Commentaires de l'Apocalypse de Beatus de Liébana

Les dragons des plus anciennes miniatures mozarabes des Commentaires de l'Apocalypse du moine Beatus de Liébana s'ornent d'un étrange dragon au nœud en forme de cœur, une forme que l'on retrouve déjà dans les sceaux-cylindres du Proche-Orient archaïque. Ce nœud est devenu le symbole des éclipses en astrologie, et plus généralement celui de la huitième planète, dite le Dragon (al-Jawzahar), à l'époque islamique, un héritage indien et iranien que les miniaturistes chrétiens ont utilisé, peut-être fortuitement, pour leurs dragons apocalyptiques.

astrologie - Apocalypse - Beatus de Liébana - dragon - éclipse

\section{Eclipse or Apocalypse. On the Dragon Knot in the Miniatures of the Beatus of Liébana's Commentaries of the Apocalypse}

The dragons of the oldest Mozarabic miniatures of the Commentaries of the Apocalypse by the monk Beatus of Liébana are decorated with a strange dragon knot shaped as a heart, a form that already exists in cylinder seals from the archaic Middle East. This knot has become the symbol of eclipses in astrology and more generally that of the eighth planet, known in the Islamic era as the Dragon (al-Jawzahar), an Indian and Iranian legacy that Christian miniaturists have used-perhaps fortuitously - for their apocalyptic dragons.

astrology - Apocalypse - Beatus of Liébana - dragon - eclipse 


\section{Annexe}

Les manuscrits et les folios concernés sont les suivants:

1/ Beatus de la bibliothèque Pierpont Morgan (New York, Pierpont Morgan Library, 644, Tábara [?], pour San Miguel de Escalada, enlumineur Maius, vers 940-945). Le nœud apparaît dans le double fo $152 \mathrm{v}^{\circ}-153 \mathrm{r}^{\circ}$ : un immense dragon au corps brun écaillé menace la Femme revêtue de Soleil de ses sept têtes effrayantes, alors que, dans la partie inférieure de la miniature, les damnés périssent en Enfer, Dieu trône en compagnie des anges dans la partie supérieure ${ }^{131}$ (planche 1).

2/ Beatus de Valladolid (Valladolid, Bibliothèque de l'Université, 433, León, Valcavado?, Obeco, 970) ${ }^{132}$, dans l'épisode de l'adoration de la Bête (Apoc. XII : 1-18), le dragon possède, à proximité des sept têtes, un énorme nœud en forme de cœur ( $\mathrm{f}^{\mathrm{o}}$ $134 \mathrm{v}^{\mathrm{o}}-135 \mathrm{r}^{\mathrm{o}}$, planche 2); dans le règne de la bête à sept têtes au fo $130 \mathrm{v}^{\mathrm{o}}-131 \mathrm{r}^{\mathrm{o}}$ (Apoc. XIII : 1-10, planche 3); la bête vomissant des grenouilles au fo $157 \mathrm{r}^{\circ}$ (planche 4).

3/ Beatus de Girona (Girona, Musée de la Cathédrale, inv. 7 [11], Tábara, Emeritus, 975) ${ }^{133}$, les démons crachent des esprits en forme de grenouilles (Apoc. XVI: 13-16, fo $204 v^{\circ}$, planche 5).

4/ Beatus de la Seú d’Urgell (Urgell, Musée diocésain de la Seú d’Urgell, s. inv. 501 , León, fin $x^{\mathrm{e}} \mathrm{s}$.) ${ }^{134}$. Trois miniatures à nœuds: dans l'épisode de la femme revêtue de Soleil, un nœud précède les sept têtes (Apoc. XII: 1-18, fo $140 \mathrm{v}^{\mathrm{o}}-141 \mathrm{r}^{\mathrm{o}}$, planche 6$)^{135}$; dans l'adoration de la Bête (Apoc. XIII : 1-10, fo $144 \mathrm{v}^{\circ}-145 \mathrm{r}^{\circ}$, planche 7); dans le dragon vomissant des grenouilles (Apoc. XVI: $13-16, \mathrm{f}^{\circ} 164 \mathrm{v}^{\mathrm{o}}$, planche 8 ).

5/ Beatus de Madrid (Madrid, Bibliothèque nationale, VIT 14-2, León, seconde moitié du $\mathrm{X}^{\mathrm{e}} \mathrm{s}$.) ${ }^{136}$ qui nous offre trois visions de ce nœud: un dragon immense, pourvu comme la bête à cornes qui lui fait face de sept têtes à gueules béantes, ondule son corps rouge à ventre blanc, piqueté de points rouges et noirs, et au milieu duquel se détache un entrelacs ou nœud en forme de cœur (fo $186 \mathrm{v}^{\circ}-187 \mathrm{r}^{\circ}$, planche 9); dans la scène de l'adoration de la Bête aux Sept Têtes ( $f^{\circ} 191 v^{o}$, planche 10$)^{137}$; enfin, dans la miniature

131. J. Williams, The Illustrated..., vol. I, p. 11; vol. II, pl. 4 et 5 et p. 21-33 et fig. 62. Voir aussi J. Williams, B. A. Shailor, A Spanish Apocalypse, the Morgan Beatus Manuscript, New York, 1991.

132. J. Williams, The Illustrated..., vol. I, p. 11, ill. 2, no 193 et 194 et fig. 207 et 38-42. Voir le fac-similé G. Roura i Güibas, C. Miranda García-Tejedor, Beato de Liébana de Girona, Girona, Museo Diocesano, núm. inv. 7 (11), Barcelone, 2003.

133. J. Williams, The Illustrated..., vol. II, p. 51-64, pl. 361.

134. A. CAgigós Soro, El Beato de la Seu d'Urgell y todas sus miniaturas : un libro del primer milenio con mensajes para hoy, Urgell, 2001, pl. 48, p. 142-143; pl. 49, p. 147, pl. 64, p. 169 et P. K. KLeIN, Beatus de Liébana, Codex Urgellensis, comentario a la edición facsimi, trad. P. DE LA RIESTRA, Torrejón de Ardoz, 2002.

135. J. Williams, The Illustrated..., vol. III, p. 17-20, fig. 53-54, fig. 54-55 et 69.

136. Voir le splendide fac-similé édité par J. Yarza Luaces, M. Sánchez Mariana, Beato de Liébana. Códice de Fernando I y Doña Sancha. Madrid, Biblioteca Nacional, vitr. 14-2, Barcelone, 1994.

137. J. Williams, The Illustrated..., vol. III, p. 160, p. 35-41, fig. 295-296, fig. 297, fig. 315. J. Yarza Luaces, M. Sánchez Mariana, Beato de Liébana, vol. 1, p. 171-177, p. 190-192. 
montrant la bouche du dragon vomissant des bêtes immondes ${ }^{138}\left(\mathrm{f}^{\circ} 220 \mathrm{v}^{\circ}\right)$, le corps du dragon se pare une fois encore dans la partie supérieure d'un nœud en forme de cœur (planche 11).

6/ L'un des dragons du Beatus de Saint-Sever ${ }^{139}$ (Paris, BnF, lat. 8878, Saint-Sever sur l'Adour, copiste Stephanus Garsia Placidus et al., troisième quart du XI ${ }^{\mathrm{e}} \mathrm{s} ., \mathrm{f}^{\circ} 202 \mathrm{v}^{\circ}$ ) n'échappe pas à ce motif dans la scène où Satan apparaît vaincu et enchaîné, et le dragon tenu en laisse par saint Pierre (planche 12).

7/ Dans le Beatus d'Osma (Burgo de Osma, Archives de la Cathédrale, Codex 1, janvier 1086, Sahagún, scribe Petrus et enlumineur Martinus), on repère un nœud dans la miniature présentant la femme couronnée de Soleil, fo $147 \mathrm{v}^{\circ}$-148 (planche 13) ${ }^{140}$.

8/ The Silos Beatus ${ }^{141}$ (Londres, British Library, Add. 11695), 18 avril 1091 et juillet 1109, Santo Domingo de Silos, Munnio, Domingo de Silos et enluminé par Petrus, $f^{\circ} 178 v^{\circ}$. Là encore, le nœud apparaît dans le dragon qui vomit des esprits en forme de grenouilles (planche 14).

9/ Dans le Beatus de Manchester (Manchester, John Rylands Library, Lat. 8, fo $14 \mathrm{r}^{\circ}$, Burgos, San Pedro de Cardeña, daté 1175$)^{142}$. Une scène «non apocalyptique», l'oiseau terrassant le serpent, reproduit l'histoire d'Ichneumon l'Égyptien luttant contre le serpent, thème de la littérature classique véhiculé par le Physiologus qui introduit en Europe, à l'image d'autres manuscrits byzantins, de nouveaux thèmes iconographiques ${ }^{143}$. Le corps du reptile s'enroule en un nœud particulier (planche 15). Le dragon vomissant des esprits en forme de grenouilles a lui aussi ce nœud (fo 171 , planche 16) ${ }^{144}$.

10/ Dans la représentation de Babylone entourée de serpents du Beatus de Las Huelgas ${ }^{145}$ (New York, Pierpont Morgan Library, ms. 429, Burgos ou Tolède, septembre 1220), on peut observer dans une des visions de Daniel, Babylone aux serpents, deux nœuds parant le corps de chacun des serpents encerclant Babylone (planche 17).

138. F. M. Ricci, U. Eco, Beatus de Liébana, Miniatures du Beatus de Ferdinand I et Sanche, Madrid, B.N., Vit. 14-2, Milan/Paris, 1982, p. 132.

139. J. Williams, The Illustrated..., vol. III, p. 44-58, fig. 458. Illustrations dans H. GarCiA ArÀEz Ferrer, La miniatura en los codices de Baeto de Liébana, Su tradicion pictorica, Madrid, 1992, p. 31 et Beato de LiéBana, Commentaires sur l'Apocalypse et le Livre de Daniel, éd. en facsim. du ms. de l'abbaye de Saint-Sever, conservé à la Bibliothèque nationale de Paris sous la cote Ms. lat. 8878, Madrid, 1984.

140. J. Williams, The Illustrated..., vol. IV, p. 18-26, fig. 284a/b.

141. Ibid., vol. IV, p. 31-41, fig. 302. M. C. Vivancos, Beato de Liébana, Códice del Monasterio de Santo Domingo de Silos, London, British Library, Add.ms. 11695, Barcelone, 2004.

142. J. Williams, The Illustrated..., vol. V, p. 19-24, 11 et pl. 33 : Manchester, John Rylands Library, ms. lat. 8, 1175, Burgos, San Pedro de Cardeña (?), P. K. KLein, Beato de Liébana: la ilustración de los manuscritos de Beato y el códice de Manchester, Valence, 2002, p. 39 et p. 60-63.

143. R. WittKower, L'Orient fabuleux..., p. 33. R. WitTKower, «Miraculous Birds, Physiologus in Beatus Manuscripts», Journal of the Warburg and Courtauld Institutes, 1 (1937), p. 253-254, pl. 33 .

144. J. Williams, The Illustrated..., vol. V, fig. 96.

145. M. Mentré, «Damas et Babylone», Le Monde de la Bible, 98 (1996), p. 32 et J. Williams, The Illustrated..., vol. V, p. 38-42, fig. 430. 
11/ Beatus, dit «Arroyo Beatus», datant du début du XIII ${ }^{\mathrm{e}}$ s., peut-être réalisé à San Pedro de Cardeña (Paris, BnF, n.a.1. 2290, fo $\left.110 v^{\circ}-111 r^{\circ}\right)^{146}$. Dans la scène de la femme vêtue de Soleil, la Bête menacée par l'ange porte aussi un nœud dans sa queue (planche 18).

Par ailleurs, nous retrouvons fréquemment ce motif du nœud en forme de cœur dans d'autres Beatus et en thème décoratif des enluminures de certaines miniatures : par exemple, dans le Beatus de Girona au fo 19, l'alpha d'ouverture présente une variante de ce motif sous toutes ses formes ${ }^{147}$; on le retrouve dans le décor du pourtour de la miniature présentant la croix d'Oviedo dans l'Antiphonaire de la cathédrale de León ${ }^{148}$, et dans bon nombre de lettres enluminées ${ }^{149}$. Notons que les manuscrits concernés sont identifiés dans les stemma de John Williams comme étant les plus anciens et constituant des familles bien identifiées, soit : le manuscrit 2 d'où sont issus les $8,4,11$ (très proches) et le 16 par ailleurs; d'autre part sont visiblement apparentés les $6,24,25$ et $20^{150}$.

146. J. Williams, The Illustrated ..., vol. V, p. 48-58. M. C. Vivancos, D. Ocón, C. Bernis et al., Beato de Liébana : códice del monasterio cisterciense de San Andrés de Arroyo, Barcelone, 1998, p. 250-251.

147. Beatus de Gérone, Cathédrale, 7, fo 19, 975, Espagne, Alpha d'ouverture, M. Mentré, La Peinture mozarabe, un art chrétien hispanique autour de l'an 1000, Paris, 1995, pl. 1.

148. Antiphonaire, León, Cathédrale, $8, \mathrm{f}^{\circ} 5 \mathrm{v}^{\circ}$ et f $\mathrm{f}^{\mathrm{o}} 68 \mathrm{r}^{\circ}, \mathrm{x}^{\mathrm{e}}$ et $\mathrm{XI}^{\mathrm{e}} \mathrm{s}$., M. Mentré, La Peinture..., pl. 5 et 12.

149. Hildefonsus Toledanus, De Virginitate sanctae Mariae, Paris, BnF, lat. 2855, fo $73 \mathrm{v}^{\circ}$, Albelda, vers 951 (M. Mentré, La Peinture..., pl. 6) ; Vitae Sanctorum, Paris, BnF, n.a.lat. 2178, $\mathrm{f}^{\mathrm{o}} 130 \mathrm{v}^{\mathrm{o}}$, seconde moitié du $\mathrm{x}^{\mathrm{e}}$ s., León ou Castille (ibid., pl. 10); Vitae sanctorum, Madrid, B.N., 822, fo $22 \mathrm{v}^{\mathrm{o}}$, seconde moitié du $\mathrm{x}^{\mathrm{e}}$ s. (ibid., pl. 43); Biblia primera, León, Saint Isidore, 2, 960, fo $181 \mathrm{r}^{\circ}$, $\mathrm{X}^{\mathrm{e}} \mathrm{s} ., \mathrm{pl} .43$.

150. J. Williams, The Illustrated..., vol. I, stemma C, p. 14. Il est notable que toute une série de copies des «Beatus» ne comportent pas de nœud, celui de San Millán de la Cogolla par exemple, Soledad de Silva y Verástegui, La miniatura en el Monasterio de San Millán de la Cogolla : una contribución al estudio de los códices miniados en los siglos XI al XIII, Logroño, 1999, Madrid, B.A.H., cod. 33, p. 64-113. 


\section{ILLUSTRATIONS}

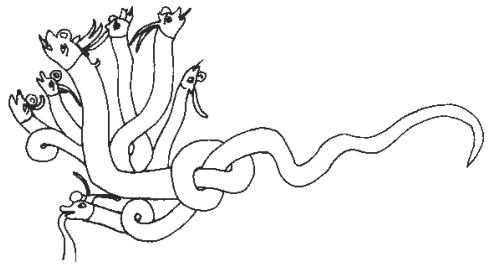

Planche 1 - Beatus in Apocalypsin, New York, Pierpont Morgan Library, ms. 644, Tábara (?), pour San Miguel de Escalada, enlumineur Maius, vers 940-945, fo $^{\circ} 152 \mathrm{v}^{\mathrm{o}}-153 \mathrm{r}^{\circ}$.

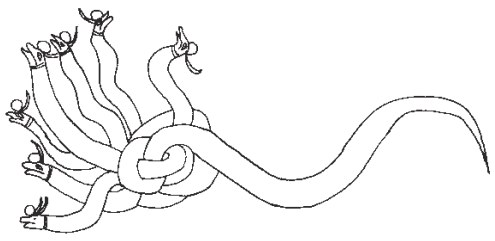

Planche 3 - Beatus in Apocalypsin, Valladolid, Bibliothèque de l'Université, ms. 433, fo $130 v^{\circ}-131 r^{\circ}, 970$, León, Valcavado (?), enlumineur Obeco.

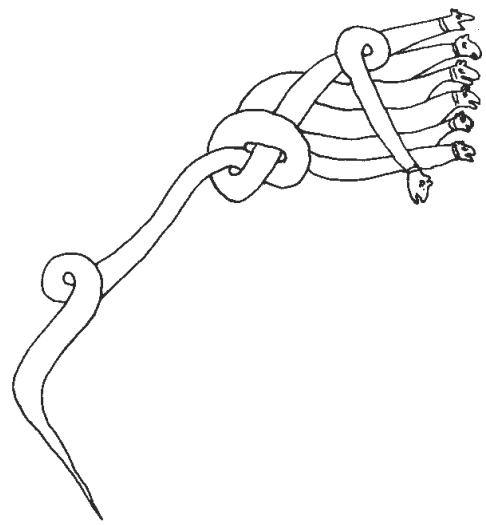

Planche 2 - Beatus in Apocalypsin, Valladolid, Bibliothèque de l'Université, ms. 433, 970, León, Valcavado (?), enlumineur Obeco, $\mathrm{f}^{\mathrm{o}} 134 \mathrm{v}^{\mathrm{o}}-135 \mathrm{r}^{\circ}$.

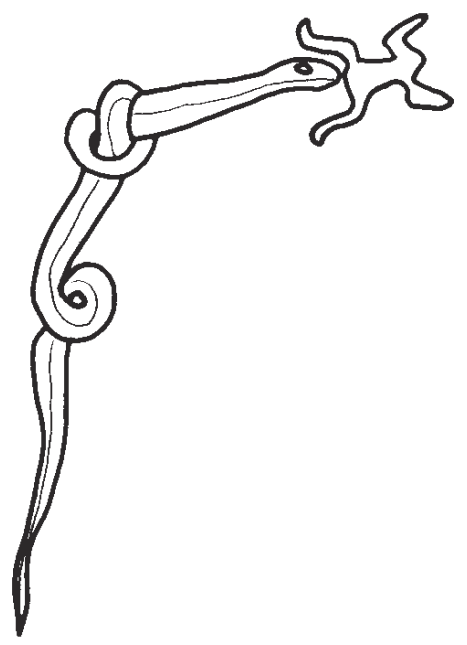

Planche 4 - Beatus in Apocalypsin,

Valladolid, Bibliothèque de l'Université, ms. 433, fo $157 r^{\circ}, 970$, León,

Valcavado (?), enlumineur Obeco. 


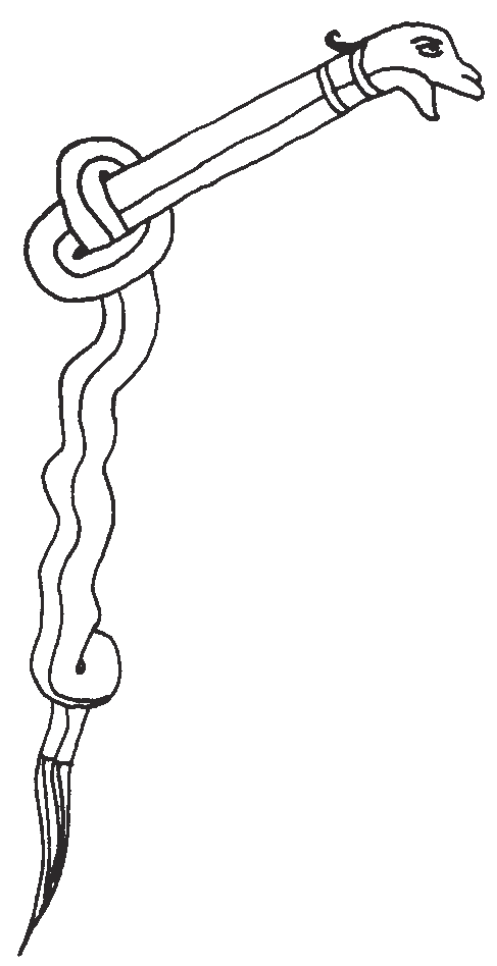

Planche 5 - Beatus in Apocalypsin, Girona, Musée de la Cathédrale, ms. inv. 7 (11), fo $204 v^{\circ}, 975$, Tábara, Emeritus.

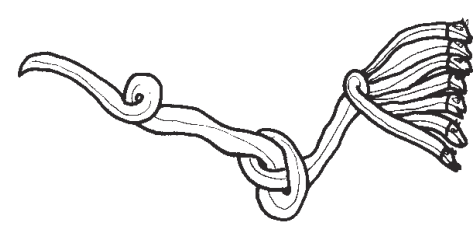

Planche 7 - Beatus in Apocalypsin, Urgell, Musée diocésain de la Seú d'Urgell, ms. $\mathrm{n}^{\circ}$ inv. 501, fo $144 \mathrm{v}^{\circ}-145 \mathrm{r}^{\circ}$, fin $\mathrm{X}^{\mathrm{e}}$ siècle.

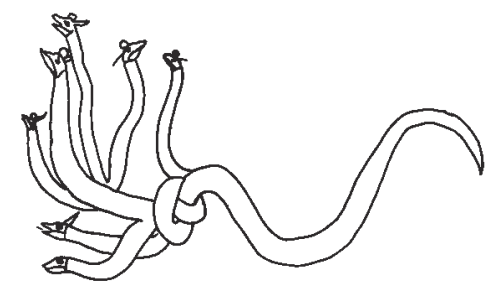

Planche 6 - Beatus in Apocalypsin, Urgell, Musée diocésain de la Seú d'Urgell, ms. ${ }^{\circ}$ inv. 501, fo $140 \mathrm{v}^{\circ}-141 \mathrm{r}^{\circ}$, fin $x^{e}$ siècle.

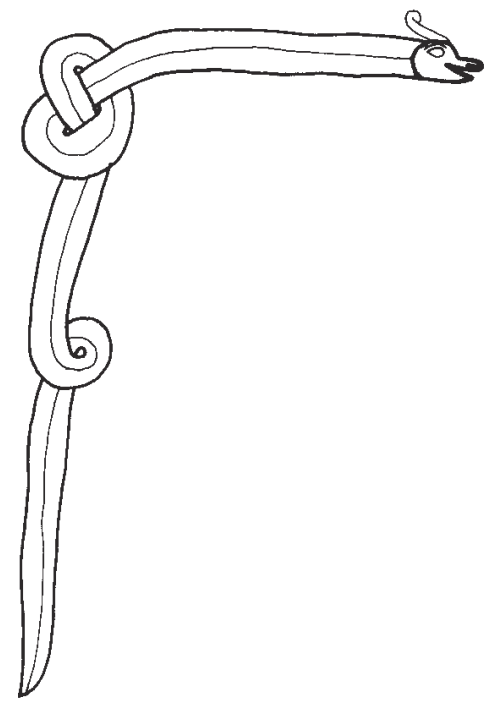

Planche 8 - Beatus in Apocalypsin, Urgell, Musée diocésain de la Seú d'Urgell, ms. $\mathrm{n}^{\circ}$ inv. 501, fin $\mathrm{x}^{\mathrm{e}}$ siècle, Le dragon vomissant des grenouilles, fo $164 v^{\circ}$. 


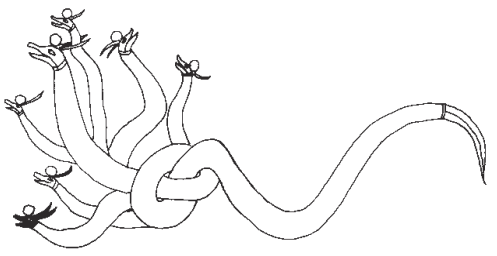

Planche 9 - Beatus in Apocalypsin,

Madrid, Bibliothèque nationale, ms. VIT. 14-2, León, seconde moitié du $\mathrm{X}^{\mathrm{e}}$ siècle, $f^{\circ} 186 v^{\circ}-187 r^{\circ}$.

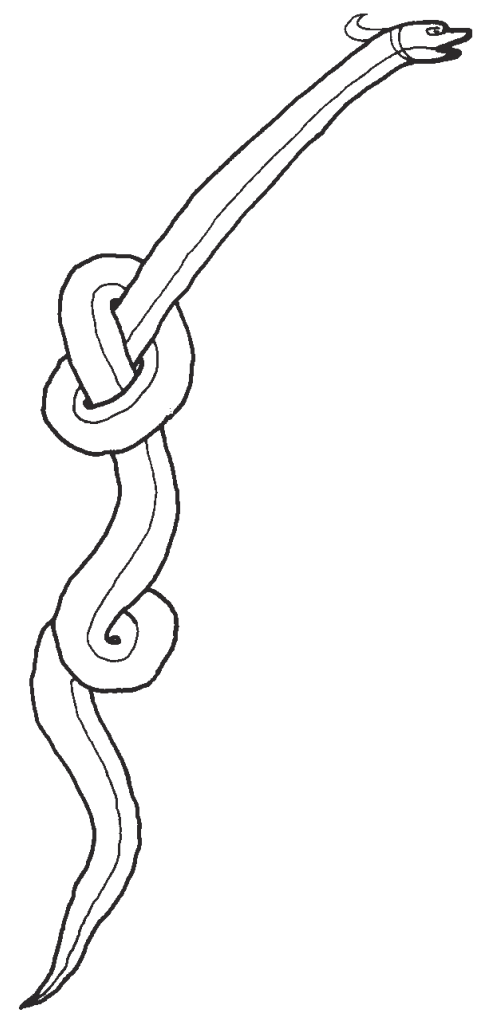

Planche 11 - Beatus in Apocalypsin,

Madrid, Bibliothèque nationale, ms. VIT 14-2, fo $220 v^{\circ}$, León, seconde moitié du $\mathrm{X}^{\mathrm{e}}$ siècle.

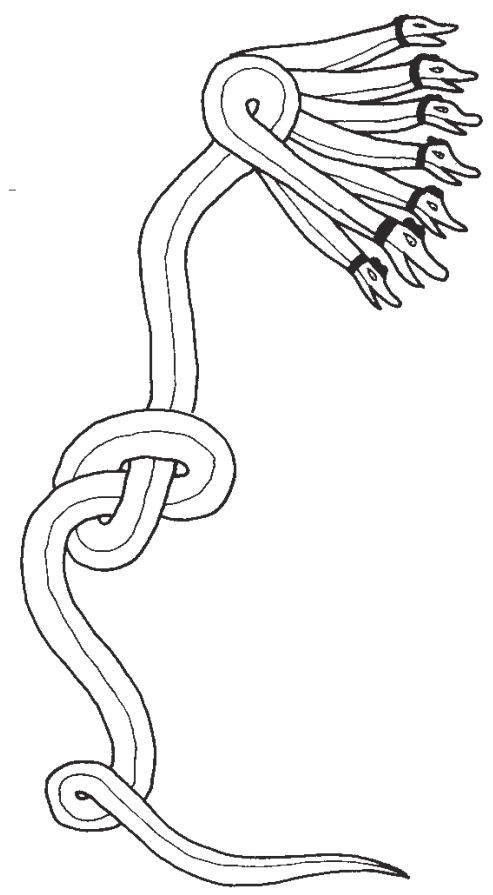

Planche 10 - Beatus in Apocalypsin,

Madrid, Bibliothèque nationale, ms. VIT. 14-2, fo $191 v^{\circ}$, León, seconde moitié du $\mathrm{X}^{\mathrm{e}}$ siècle.

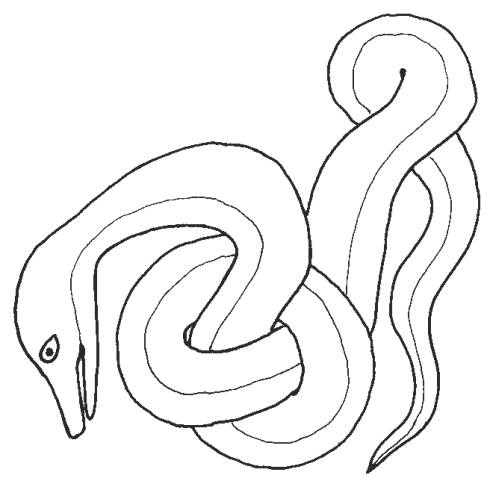

Planche 12 - Beatus in Apocalypsin, Paris, BnF, Ms. Lat. 8878, fo $202 v^{\circ}$, troisième quart du $\mathrm{XI}^{\mathrm{e}}$ siècle, Saint-Sever sur l'Adour, Stephanus Garsia Placidus et al. 


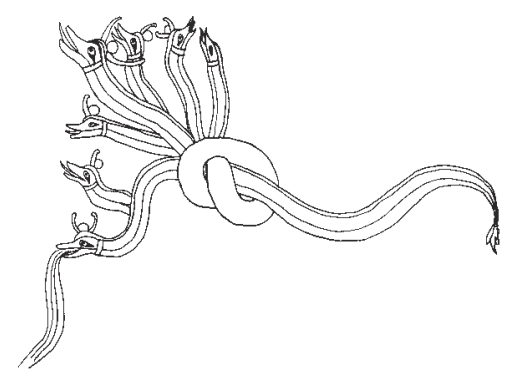

Planche 13 - Beatus in Apocalypsin, Burgo de Osma, Archives de la Cathédrale, Codex $1, \mathrm{f}^{\circ} 147 \mathrm{v}^{\circ}-148 \mathrm{r}^{\circ}$, janvier 1086, Sahagún, scribe Petrus et enlumineur Martinus.

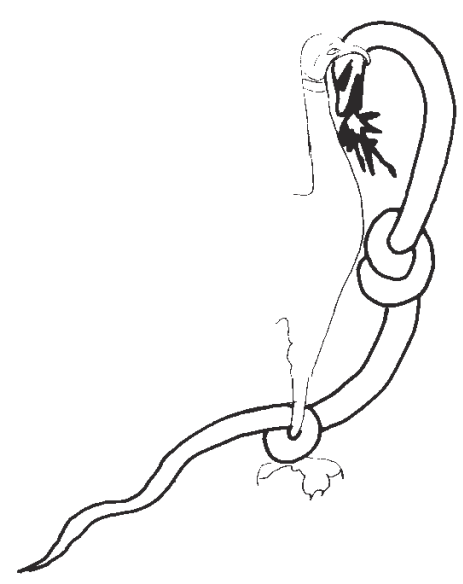

Planche 15 - Beatus in Apocalypsin, Manchester, John Rylands Library, ms. lat. $8, \mathrm{f}^{\circ} 14 \mathrm{r}^{\circ}$, Burgos, San Pedro de Cardeña, daté 1175 .
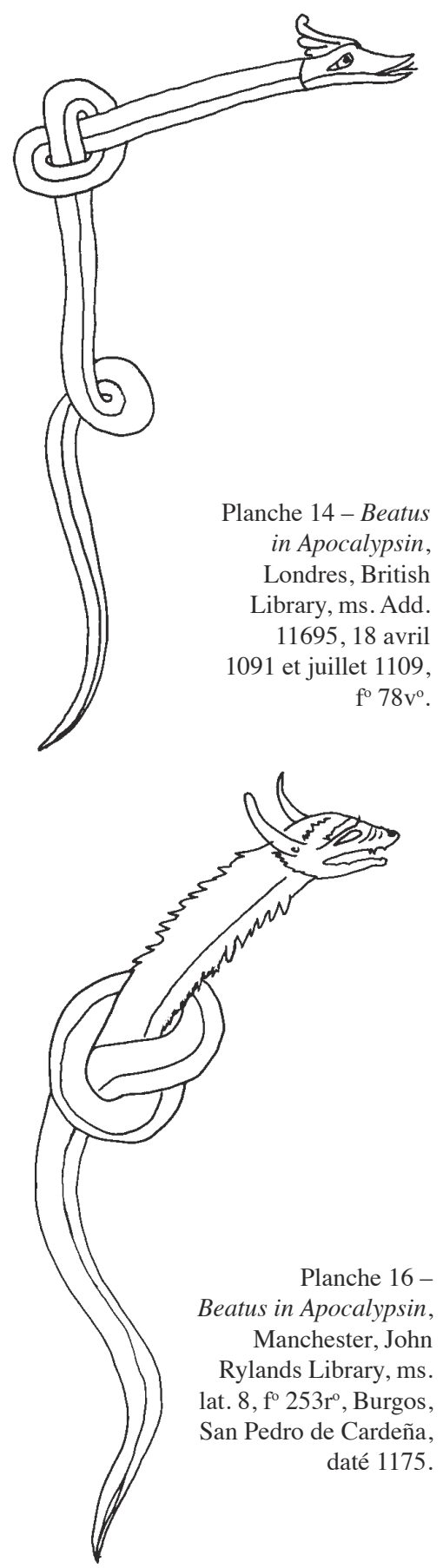


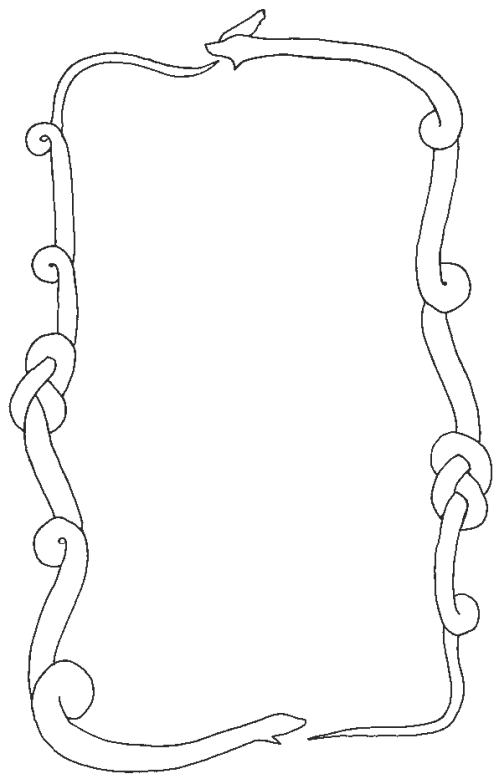

Planche 17 - Babylone (Dan. I) : Beatus in Apocalypsin, New York, Pierpont Morgan Library, ms. 429, fo $147 \mathrm{r}^{\circ}$, Las Huelgas, Burgos ou Tolède, septembre 1220 .

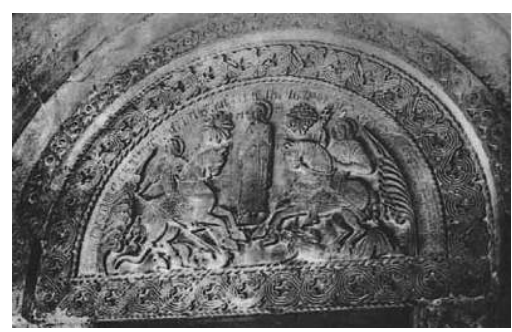

Planche 19 - Tympan ouest de l'église de Nicorzminda, Géorgie, XIII ${ }^{\mathrm{e}}$ siècle, photographie dans J. Baltrusaitis, L'Art grégorien et arménien, Paris, 1929, pl. 67.

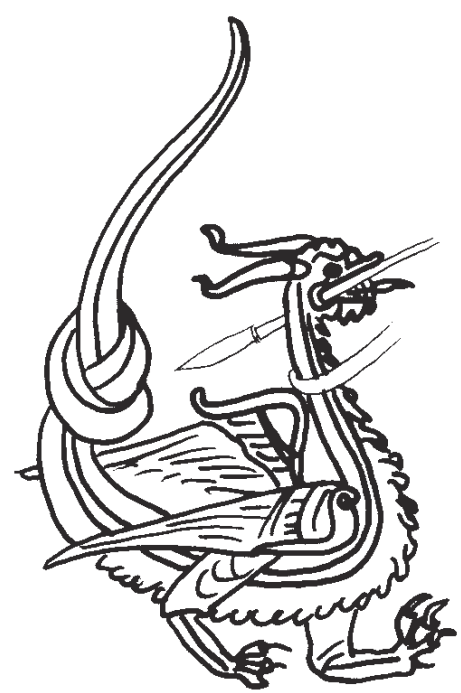

Planche 18 - Beatus in Apocalypsin, Arroyo Beatus, Paris, BnF, ms. n.a.l. $2290, f^{\circ} 110 v^{\circ}-111 r^{\circ}$, début du XIII ${ }^{\mathrm{e}}$ siècle, San Pedro de Cardeña.

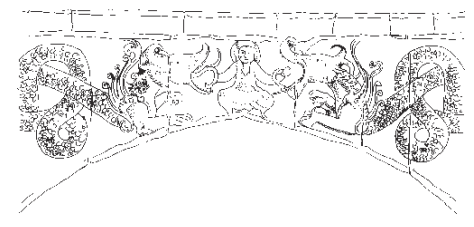

Planche 20 - Thèmes décorant la Porte du Talisman à Bagdad érigée par le calife al-Nâsir en 1221.

Illustration Pl. 26, 28 d'après F. Sarre, Der Kiosk von Konia, Berlin, 1936, Verlag für Kunstwissenschaft. 


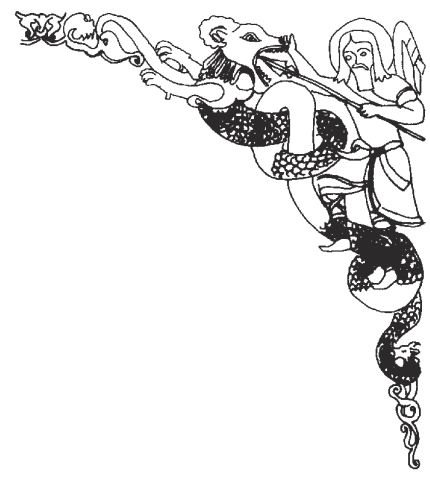

Planche 21 - Un saint luttant contre un dragon sur le bas-relief sculpté d'un caravansérail de Sinjar, près de Mossoul, milieu XIII ${ }^{\mathrm{e}}$ siècle, illustrations pl. 25, 26 d'après F. Sarre, Der Kiosk von Konia, Berlin, 1936.
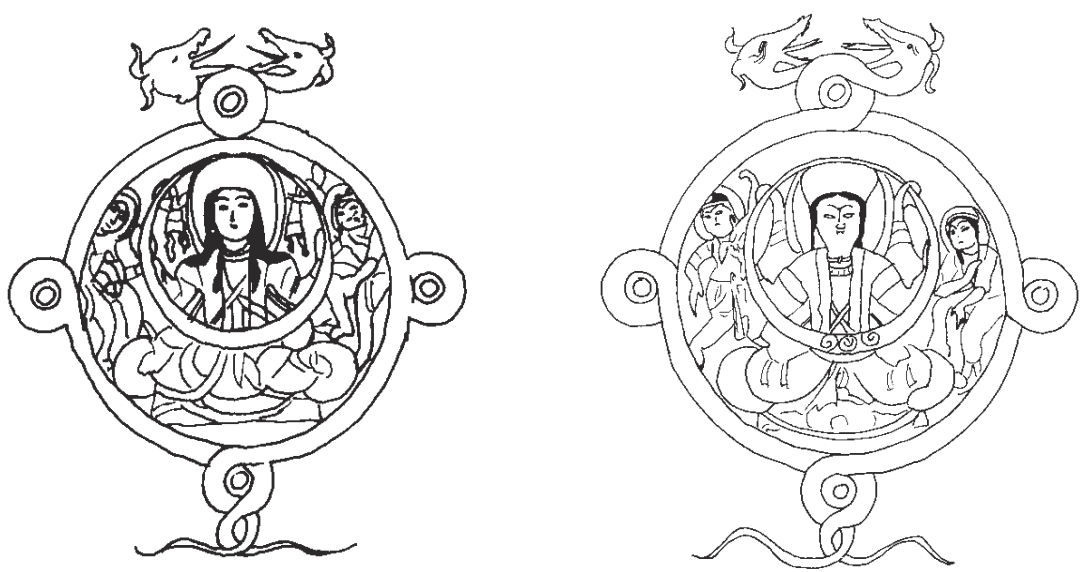

Planche 22 - Double frontispice du Kitâb al-Diryâq, Paris, BnF, ms. ar. 2964, 1199, Mossoul [?]. 


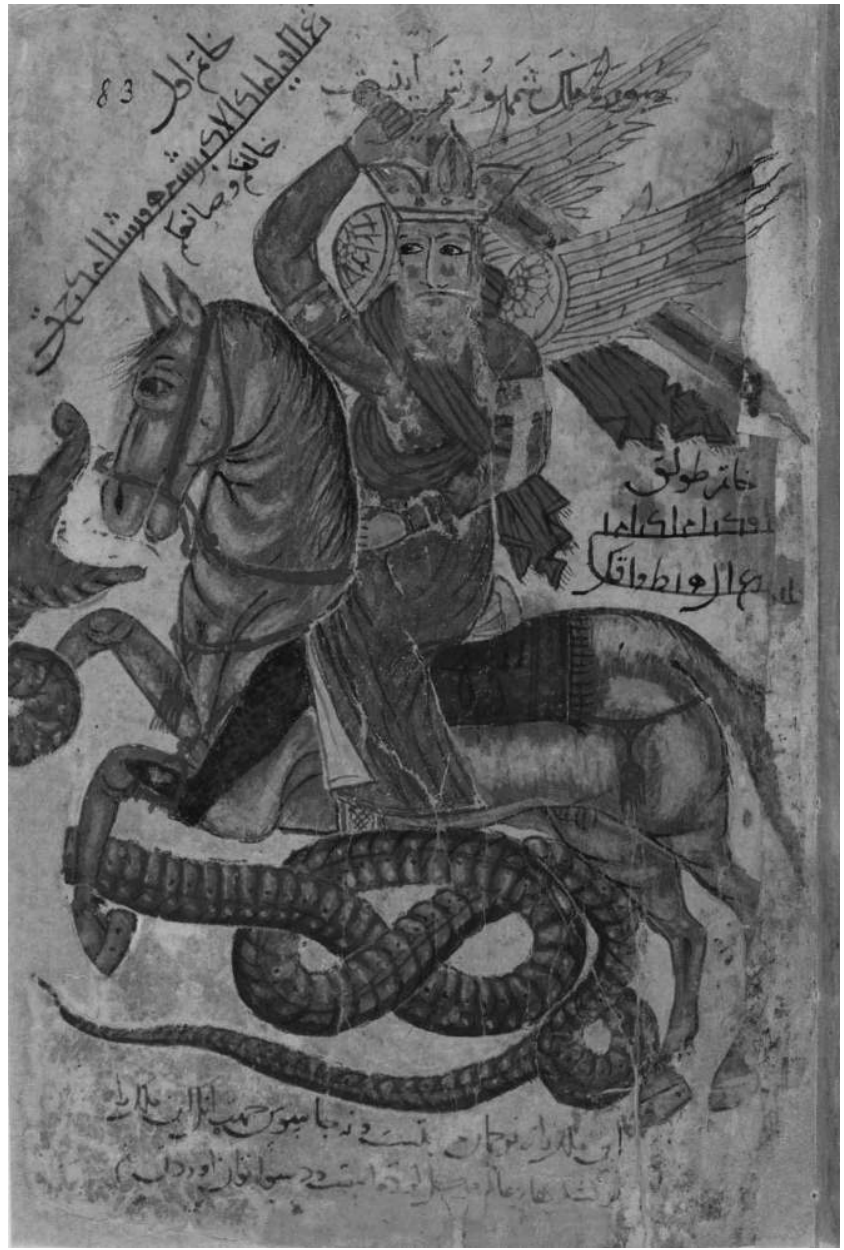

Planche 23 - L'ange Shamhûraš dans le Daqấ'iq al-Haqâ'iq (Paris, BnF, ms. persan 174, Aqsarây, 1272, fo $83 \mathrm{r}^{\circ} 16 \times 21 \mathrm{~cm}$ ). 